\title{
Glioma cells are resistant to inflammation-induced alterations of mitochondrial dynamics
}

\author{
WANGE FAN ${ }^{1 *}$, YANAN SONG $^{1 *}$, ZONGYAO REN $^{1,2^{*}}$, XIAOLI CHENG ${ }^{1 *}$, \\ PU LI ${ }^{1}$, HUILING SONG ${ }^{1}$ and LIYUN JIA ${ }^{1}$ \\ ${ }^{1}$ Department of Medical Genetics and Cell Biology, School of Basic Medical Sciences, \\ Zhengzhou University, Zhengzhou, Henan 450001; ${ }^{2}$ Center for Medical Genetics, \\ School of Life Sciences, Central South University, Changsha, Hunan 410012, P.R. China
}

Received June 19, 2020; Accepted September 24, 2020

DOI: $10.3892 /$ ijo.2020.5134

\begin{abstract}
Accumulating evidence suggests that inflammation is present in solid tumors. However, it is poorly understood whether inflammation exists in glioma and how it affects the metabolic signature of glioma. By analyzing immunohistochemical data and gene expression data downloaded from bioinformatic datasets, the present study revealed an accumulation of inflammatory cells in glioma, activation of microglia, upregulation of proinflammatory factors (including $I L-6, I L-8$, hypoxia-inducible factor-1 $\alpha$, STAT3, NF- $\mathrm{B} 1$ and $N F-\kappa B 2$ ), destruction of mitochondrial structure and altered expression levels of electron transfer chain complexes and metabolic enzymes. By monitoring glioma cells following proinflammatory stimulation, the current study observed a remodeling
\end{abstract}

Correspondence to: Dr Liyun Jia, Department of Medical Genetics and Cell Biology, School of Basic Medical Sciences, Zhengzhou University, 100 Kexue Avenue, Zhengzhou, Henan 450001, P.R. China

E-mail: liyunjia1982@163.com

"Contributed equally

Abbreviations: HIF-1 $\alpha$, hypoxia-inducible factor- $1 \alpha$; HMGB1, high-mobility group box 1 protein; Iba1, ionized calcium binding adapter molecule 1; LAMP1, lysosomal associated membrane protein 1; SDHB, iron-sulfur protein subunit of succinate dehydrogenase; COX6B1, cytochrome $\mathrm{C}$ oxidase subunit VIb; HK1, hexokinase 1; CS, citrate synthase; LDHA, lactate dehydrogenase A; ROS, reactive oxygen species; ceROS, cellular ROS; mtROS, mitochondrial ROS; ETC, electron transfer chain; OXPHOS, oxidative phosphorylation; TCA, tricarboxylic acid; $\Delta \Psi \mathrm{m}$, mitochondrial membrane potential; HNE, 4-hydroxynonenal; DCFH-DA, 2',7'-dichlorodihydrofluorescein diacetate; LPS, lipopolysaccharide; para-NT, paraneoplastic tissues; LGG, low-grade glioma; GBM, glioblastoma; IRS, immunoreactive score; CGGA, Chinese Glioma Genome Atlas; TCGA, The Cancer Genome Atlas

Key words: apoptosis, glioma, inflammation, metabolic reprogramming, mitochondrial network, mitophagy of their mitochondrial network via mitochondrial fission. More than half of the mitochondria presented ring-shaped or spherical morphologies. Transmission electron microscopic analyses revealed mitochondrial swelling with partial or total cristolysis. Furthermore, proinflammatory stimuli resulted in increased generation of reactive oxygen species, decreased mitochondrial membrane potential and reprogrammed metabolism. The defective mitochondria were not eliminated via mitophagy. However, cell viability was not affected, and apoptosis was decreased in glioma cells after proinflammatory stimuli. Overall, the present findings suggested that inflammation may be present in glioma and that glioma cells may be resistant to inflammation-induced mitochondrial dysfunction.

\section{Introduction}

Glioma is a primary brain tumor that originates histologically from normal glial cells or precursor cells, accounting for $>70 \%$ of malignant brain tumors in the United States in 2009-2013, and it is the most prevalent primary tumor of the brain and spinal cord (1). Glioma is a lethal tumor characterized by diffuse infiltration in the brain and high resistance to conventional cancer therapies (1). Despite relevant progress in conventional treatments, the prognosis in patients with glioma remains almost invariably dismal.

The genesis of gliomas is a complex, multistep process that includes cellular neoplastic transformation, resistance to apoptosis, loss of cell cycle control, angiogenesis and the acquisition of invasive properties (2). Among a number of different biomolecular events, associations between inflammation and the development of this type of cancer have been demonstrated (2,3). As a self-limiting process, the acute inflammatory response can be anti-tumorigenic and serve a role in tumor suppression (4). However, chronic inflammation frequently leads to various chronic diseases, including cancer $(5,6)$, and is associated with various phases of tumorigenesis, including cell proliferation, neoplastic transformation, apoptosis evasion, loss of cell cycle control, angiogenesis and metastasis $(7,8)$. During the process of chronic inflammation, a number of proinflammatory molecules can be synthesized by resident brain cells and lymphocytes that invade the affected brain tissue, such as IL-6, IL-1 $\beta$, TNF $\alpha$, inducible nitric oxide synthase, 
hypoxia-inducible factor-1 $\alpha$ (HIF-1 $\alpha)$, STAT3, chemokines and prostaglandins (2,7). The collective activity of these molecules is largely responsible for the pro-tumorigenic effects (9). In addition, free radicals and aldehydes generated during chronic inflammation, such as reactive oxygen species (ROS) and reactive nitrogen species, can induce DNA damage and increase the deleterious DNA mutation rate $(7,10)$. Accordingly, chronic inflammation is a major cause of cancer (11).

Mitochondria serve key roles in cellular energy metabolism, generation of free radicals and apoptosis (12). They are biosensors that allow cells to adapt to environmental stress (13). Since tumorigenesis requires flexibility for the tumors to adapt to cellular and environmental alterations, it is not surprising that mitochondria serve a key role in this process (14). Mitochondrial abnormalities have been recognized in numerous types of tumor, such as endometrial cancer (14), cervical cancer (15), breast cancer (16), epithelial ovarian cancer (17) and glioma (18). Mitochondrial dysfunction in glioma involves abnormalities in energy metabolism, changes in mitochondrial membrane potential $(\Delta \Psi \mathrm{m})$ regulation, disruption of apoptotic signaling pathways $(19,20)$ and mutations in the tricarboxylic acid (TCA) cycle enzyme isocitrate dehydrogenase $(21,22)$. The characterization of the full extent of mitochondrial abnormalities in glioma is a rapidly expanding area of investigation. In tumors, the most common mitochondria-associated effect is known as the 'Warburg effect', during which cancer cells fuel mitochondrial respiration via aerobic glycolysis rather than via complete glucose oxidation (23). Otto Warburg suggested that this phenomenon may be caused by an impaired mitochondrial respiratory capacity in these cells (24). In addition to energy production, mitochondria perform numerous other roles, such as ROS generation, production of redox molecules and metabolites, and regulation of their own biogenesis and turnover, and fission and fusion dynamics, as well as influencing cell signaling and cell death (25). These multifaceted functions of mitochondria impart the tumor cells with considerable flexibility for growth and survival under harsh conditions, including hypoxia, cancer treatment and nutrient depletion (25). Furthermore, cells have developed mitochondrial quality control mechanisms (mitophagy) that clear damaged mitochondria caused by pathological conditions; whether mitophagy is beneficial or harmful to cancer depends on tumor type and stage (26). During tumorigenesis, decreased mitophagy may lead to the persistence of damaged mitochondria in cells, which may result in higher levels of tumor-promoting ROS or other mitochondrial signals (25). By contrast, established tumors may require mitophagy for stress adaptation and survival $(25,27,28)$. Despite the multitude of mechanisms that have been proposed to explain the mitochondrial dynamics in tumor cells, the effects of inflammation on this cellular event in glioma remain uncharacterized. The present study aimed to explore the inflammation in glioma using glioma cell lines, clinical samples and bioinformatics, paying particular attention to the effects of inflammation on the mitochondria dynamics in glioma.

\section{Materials and methods}

Histological samples. A total of 24 paraffin-embedded glioma tissue sections (5- $\mu$ m-thick; 12 males and 12 females; age range, 36-74 years; median age of patients, 50.3 years) and 3 normal brain tissue sections (derived from decompression operations; 5 - $\mu \mathrm{m}$-thick) were obtained between January 2017 and December 2018 from the Department of Histology of the 988 Hospital of Joint Logistic Support Force (Zhengzhou, China). All samples were fixed with $4 \%$ paraformaldehyde for $24 \mathrm{~h}$ at $4{ }^{\circ} \mathrm{C}$ and evaluated by the experienced clinical pathologists at the aforementioned hospital according to the 2016 World Health Organization classification (29). Among the 24 glioma samples, 16 samples of paraneoplastic tissues (para-NT; $\sim 2 \mathrm{~cm}$ away from the tumor) were also collected. Additionally, one case of glioblastoma tissue (evaluated according to the aforementioned classification; male; 66 years) derived from the Department of Neurosurgery of the aforementioned hospital was used to perform the transmission electron microscopy (TEM) analysis. All patients had not received any other treatment prior to surgery, including radiotherapy and chemotherapy. The detailed information about the demographic parameters of patients is listed in Table SI. The present study was approved by the Life Science Ethics Committee of Zhengzhou University (Zhengzhou, China) according to the principles expressed in the Declaration of Helsinki. Written informed consent was provided by all patients with glioma. Additionally, written informed consent was obtained from the families of three patients with traumatic brain injury who underwent the decompression operations. Furthermore, mRNA microarray expression data was downloaded from the Chinese Glioma Genome Atlas (CGGA) database [http://www. cgga.org.cn; 290 adult glioma, 165 low-grade glioma (LGG) and 125 glioblastoma (GBM) samples] and The Cancer Genome Atlas (TCGA) database (https://tcga-data.nci.nih. gov; 512 adult glioma, 26 LGG and 486 GBM samples). The date of access for the two datasets was 6/30/2019, and the data downloaded from the CGGA dataset was not normalized.

Hematoxylin and eosin $(H \& E)$ staining. First, deparaffination was performed using $100 \%$ xylene (twice for $5 \mathrm{~min}$ ) and rehydrated using a gradient ethanol series (100\% twice for 5 min; 95\% twice for $5 \mathrm{~min}$; $90 \%$ for $5 \mathrm{~min}$; and $80 \%$ for $5 \mathrm{~min}$ ) at room temperature (RT). After washing with distilled water for $5 \mathrm{~min}$, the sections were stained using hematoxylin solution for $5 \mathrm{~min}$ at RT and then washed with running tap water for 1-3 sec. Subsequently, the sections were differentiated using $1 \%$ hydrochloric acid (in 70\% ethanol) for 1-3 sec. After washing with running tap water for 10-30 sec and distilled water for 1-2 sec, the sections were stained in the working eosin $Y$ solution $(0.5 \%$ in water) for $3 \mathrm{~min}$ at RT. After washing with distilled water for 1-2 sec, the sections were dehydrated using a gradient ethanol series (95\% twice for 2-3 sec and $100 \%$ twice for $2 \mathrm{~min}$ ) and then cleared with three washes of xylene ( 2 min per wash). Finally, the sections were mounted with neutral resin. Images were captured and analyzed using a light microscope (magnification, x40; IX53; Olympus Corporation).

Cell lines and reagents. The U87-MG (GBM of unknown origin) and U118-MG (GBM of unknown origin) cell lines were obtained from The Cell Bank of Type Culture Collection of the Chinese Academy of Sciences. The cell lines were authenticated via STR profiling. Cells were cultured in high-glucose DMEM (cat. no. 04-052-1ACS; Biological Industries), 
containing 10\% FBS (cat. no. 04-001-1ACS; Biological Industries) and $1 \%$ penicillin-streptomycin (cat. no. P1400; Beijing Solarbio Science \& Technology Co., Ltd.) at $37^{\circ} \mathrm{C}$ in a humidified incubator with $5 \% \mathrm{CO}_{2}$.

Reagents included lipopolysaccharide (LPS; $4 \mu \mathrm{g} / \mathrm{ml}$; cat. no. L7770; Sigma-Aldrich; Merck KGaA) and human recombinant IFN- $\gamma$ (40 ng/ml; cat. no. 11725-HNAS; Sino Biological, Inc.). Glioma cells were stimulated with LPS and IFN- $\gamma$ at $37^{\circ} \mathrm{C}$ in a humidified incubator with $5 \% \mathrm{CO}_{2}$ for 0,4 , 8 or 24 h.

Immunohistochemistry (IHC). IHC was performed on the aforementioned tissue sections. First, deparaffination was performed using $100 \%$ xylene (twice for $5 \mathrm{~min}$ ) and rehydrated using a gradient ethanol series $(100 \%$ twice for $5 \mathrm{~min}$; $95 \%$ twice for $5 \mathrm{~min}$; $90 \%$ for $5 \mathrm{~min}$; and $80 \%$ for $5 \mathrm{~min}$ ) at RT. Subsequently, antigen retrieval was performed using a citrate solution ( $\mathrm{pH} 6.0$ ) at $95^{\circ} \mathrm{C}$ for $15 \mathrm{~min}$. The endogenous peroxidase activity was quenched by incubating the slides with $3 \%$ hydrogen peroxide at RT for $30 \mathrm{~min}$, followed by washing in PBS three times for 5 min each. The sections were incubated for $1 \mathrm{~h}$ at RT with blocking solution [5\% bovine serum albumin (cat. no. A8020; Beijing Solarbio Science \& Technology Co., Ltd.) plus $0.3 \%$ Triton X-100 in PBS] and subsequently incubated at $4^{\circ} \mathrm{C}$ overnight with the following primary antibodies: Polyclonal goat anti-human ionized calcium binding adapter molecule 1 (Iba1) antibody (Abcam; cat. no. ab5076; 1:500), monoclonal mouse anti-human IL-6 antibody (Abcam; cat. no. ab9324; 1:500), polyclonal rabbit anti-human high mobility group box 1 protein (HMGB1) antibody (Abcam; cat. no. ab18256; 1:1,000), monoclonal rabbit anti-human cytochrome $\mathrm{C}$ oxidase subunit VIb (COX6B1) antibody (Abcam; cat. no. ab131277; 1:100), monoclonal mouse anti-iron-sulfur protein subunit of succinate dehydrogenase (SDHB) antibody (Abcam; cat. no. ab14714; 1:200) and polyclonal rabbit anti-human nicotinamide adenine dinucleotide (NADH) dehydrogenase subunit 6 antibody (Abcam; cat. no. ab81212; 1:400). Subsequently, the sections were rinsed with PBS and incubated with the appropriate secondary antibodies for $2 \mathrm{~h}$ at RT. For the 3,3'-diaminobenzidine (DAB) staining (HMGB1), the sections were incubated with horseradish peroxidase-conjugated goat anti-rabbit IgG polyclonal antibody (1:1,000; ZSGB-BIO, Inc.; cat. no. ZB-23) for $2 \mathrm{~h}$ at RT. Subsequently, DAB was dropped onto the slides, which were then incubated at $37^{\circ} \mathrm{C}$ for $3 \mathrm{~min}$. The sections were then counterstained using haematoxylin, washed with distilled water, differentiated using $1 \%$ hydrochloric acid (in 70\% ethanol) and mounted with neutral resin. For the fluorescence-labelled reaction, the secondary antibodies used were as follows: Fluorescein-conjugated goat anti-rabbit IgG (ZSGB-BIO, Inc.; cat. no. ZF-0311; 1:100), Alexa Fluor 488 donkey anti-rabbit IgG (Abcam; cat. no. ab150073; 1:1,000), rhodamine-conjugated goat anti-mouse IgG (ZSGB-BIO, Inc.; cat. no. ZF-0313; 1:100) and Cy3-labeled donkey anti-goat IgG (Beyotime Institute of Biotechnology; cat. no. A0502; 1:1,000). The nuclei were stained with DAPI (Beijing Solarbio Science \& Technology Co., Ltd.; cat. no. C0060; 1:5,000) at RT for 15 min. Images were acquired using an OLYMPUS confocal microscope (magnification, x40; U-TBI90; Olympus Corporation) and analyzed using Adobe Photoshop CS6 (Adobe Systems, Inc.) and Image-Pro Plus (v6.0; Media Cybernetics, Inc.). Evaluation of the staining reactions was performed based on the immunoreactive score (IRS)=staining intensity $\mathrm{x}$ percentage of positive cells (30). The staining intensity was determined as: 0 , negative; 1 , weak (light brown staining); 2 , moderate (brown staining); and 3, strong (dark brown staining). The percentage of positive cells was determined as: 0 , no positive cells; $1, \leq 10 \%$ positive cells; $2,11-50 \%$ positive cells; $3,51-80 \%$ positive cells; and $4,>80 \%$ positive cells. At least three visual fields from different areas of each tumor specimen were used for the IRS evaluation.

Immunocytochemistry (ICC). U87-MG and U118-MG glioma cells were grown on glass coverslips in 6-well plates. After the appropriate treatments with LPS $(4 \mu \mathrm{g} / \mathrm{ml})$ and IFN- $\gamma(40 \mathrm{ng} / \mathrm{ml})$ for $0,4,8$ or $24 \mathrm{~h}$ at $37^{\circ} \mathrm{C}$, cells were washed twice with ice-cold PBS and fixed with freshly prepared $4 \%$ paraformaldehyde in PBS at RT for $15 \mathrm{~min}$, followed by permeabilization with $0.3 \%$ Triton $\mathrm{X}-100$ in PBS for $20 \mathrm{~min}$. Subsequently, the cells were blocked with blocking solution (as aforementioned for IHC) for $1 \mathrm{~h}$ at RT and incubated with the following primary antibodies at $4^{\circ} \mathrm{C}$ overnight: Monoclonal mouse anti-4-hydroxynonenal (HNE) antibody (Abcam; cat. no. ab48506; 1:500), monoclonal rabbit anti-human lysosomal associated membrane protein 1 (LAMP1) antibody (Cell Signaling Technology, Inc.; cat. no. 9091; 1:200) and monoclonal rabbit anti-LC3B antibody (Cell Signaling Technology, Inc.; cat. no. 3868; 1:200). Subsequently, the cells were washed three times with PBS and stained with the appropriate fluorescently conjugated secondary antibodies (as aforementioned for IHC) for $2 \mathrm{~h}$ at RT. The nuclei were counterstained with DAPI at RT for $15 \mathrm{~min}$. In some experiments, cells were preloaded with Mitotracker Red (Invitrogen; Thermo Fisher Scientific, Inc.; cat. no. M7512; $200 \mathrm{nM}$ ) at $37^{\circ} \mathrm{C}$ for $25 \mathrm{~min}$ before fixation. Finally, the coverslips were mounted in $50 \%$ glycerol-PBS anti-fade mounting medium. Images were acquired using an OLYMPUS confocal microscope (magnification, x100) and analyzed with Adobe Photoshop CS6 and Image-Pro Plus (v6.0; Media Cybernetics, Inc.).

Evaluation of various types of mitochondria was based on the ratio between the major axis and minor axis ('Aspect' function in the 'measurements' of the Image-Pro Plus for each mitochondrion) (31). If the ratio was between 1.0 and 1.5 , the mitochondrion was categorized into the 'spherical (or ring-like)' group. If the ratio was $>1.5$, the mitochondrion was categorized into the 'rod or tubular' group (31). The ratio of cells with fragment, intermediate or tubular mitochondria was analyzed as determined by the presence of these two types of mitochondria: $>60 \%$ of spherical (or ring-like) mitochondria represented a cell with fragment mitochondria; $>60 \%$ of rod or tubular mitochondria represented a cell with tubular mitochondria; 40-60\% of both types of mitochondria represented a cell with intermediate mitochondria. A total of 50-150 cells were analyzed for each time point treatment.

To quantify the autophagic cells, the ratio of autophagic cells was analyzed as determined by the presence of LC3B puncta ( $>10$ puncta indicated a positive cell). A total of 50-150 cells were analyzed for each time point treatment. 
Cell transfection. Glioma cells were cultured to $80 \%$ confluence in sixwell culture plates, and then transfected with $2 \mu \mathrm{g}$ GFP-LC3 plasmid (provided by Professor Shuping Zhang, State Key Laboratory of Biomembrane and Membrane Biotechnology, School of Life Sciences, Tsinghua University, Beijing, China) using SimpleFect Transfection Reagent (Zhengzhou Kebang Biological Technology Co., Ltd.; cat. no. profect-01) according to the manufacturer's protocol at $37^{\circ} \mathrm{C}$ for $16 \mathrm{~h}$. Subsequent experimentations were performed $\sim 12$ h after transfection.

Western blotting. Cells were lysed in RIPA buffer (Beijing Solarbio Science \& Technology Co., Ltd.; cat. no. R0010) and incubated on ice for $\geq 30 \mathrm{~min}$. The lysates were cleared by centrifugation at $15,294 \mathrm{x} \mathrm{g}$ at $4^{\circ} \mathrm{C}$ for $15 \mathrm{~min}$, and the supernatant fractions were collected. The protein amounts in the whole-cell lysates were determined using the BCA Protein Assay kit (Beijing Solarbio Science \& Technology Co., Ltd.; cat. no. PC0020). Equal amounts of proteins (20 $\mu \mathrm{g} / \mathrm{lane})$ were separated via $10-12 \%$ SDS-PAGE, and the proteins were then transferred to PVDF membranes (EMD Millipore; cat. no. IPVH00010). Blocking was performed for $1 \mathrm{~h}$ at RT using 5\% non-fat dry milk in TBS with 1\% Tween 20 (TBST) at RT. The blots were incubated with primary antibodies overnight at $4^{\circ} \mathrm{C}$, followed by washing three times with TBST and incubation with peroxidase-conjugated goat anti-mouse or anti-rabbit IgG secondary antibodies (ZSGB-BIO, Inc.; cat. no. ZB-2305 or ZB-2301, respectively; $1: 5,000)$ for $2 \mathrm{~h}$ at RT. The primary antibodies used were as follows: Monoclonal rabbit anti-human hexokinase 1 (HK1) antibody (Cell Signaling Technology, Inc.; cat. no. 2024; 1:1,000), monoclonal rabbit anti-human citrate synthase (CS) antibody (Cell Signaling Technology, Inc.; cat. no. 14309; 1:1,000), monoclonal rabbit anti-human lactate dehydrogenase A (LDHA) antibody (Cell Signaling Technology, Inc.; cat. no. 3582; 1:1,000), monoclonal rabbit anti-human COX6B1 antibody (Abcam; cat. no. ab131277; 1:1,500), monoclonal mouse anti-SDHB antibody (Abcam; cat. no. ab14714; 1:200), monoclonal mouse anti-human $\beta$-Actin (ZSGB-BIO, Inc.; cat. no. TA-09; 1:2,000), monoclonal rabbit anti-LC3B antibody (Cell Signaling Technology, Inc.; cat. no. 3868; 1:500) and polyclonal rabbit anti-GAPDH antibody (Hangzhou Xianzhi Biological Technology Co., Ltd.; cat. no. AB-P-R 001; 1:1,000). The blots were visualized using ECL luminescence reagent (Dalian Meilun Biotechnology Co., Ltd.; cat. no. MA0186-Sep-25D) and analyzed using ImageJ v1.46r (National Institutes of Health).

TEM. The glioma tissues and the LPS- and IFN- $\gamma$-pretreated glioma cells were quickly fixed in $4 \%$ glutaraldehyde in $0.1 \mathrm{M}$ phosphate buffer ( $\mathrm{pH} 7.4$ ) at $4^{\circ} \mathrm{C}$ for $2 \mathrm{~h}$ and then harvested with a rubber scraper. After centrifugation at $106 \mathrm{x} g$ for $5 \mathrm{~min}$ at $4^{\circ} \mathrm{C}$, cell pellets immersed in $4 \%$ glutaraldehyde in $0.1 \mathrm{M}$ phosphate buffer ( $\mathrm{pH}$ 7.4) were sent to Wuhan Servicebio Technology Co., Ltd., or to the electron microscopy center of Henan University of Chinese Medicine (Zhengzhou, China) where the remaining procedures were performed. Sections were examined using a JEM-1400 electron microscope (JEOL, Ltd.).

Flow cytometric analyses. To measure the total cellular ROS (ceROS) levels, the LPS and IFN- $\gamma$-treated U87-MG cells were collected via centrifugation at $4^{\circ} \mathrm{C}$ at $106 \mathrm{x}$ g for $10 \mathrm{~min}$ and dissociated into a single-cell suspension using Accutase reagents (Beijing Solarbio Science \& Technology Co., Ltd.) according to the manufacturer's protocol. Subsequently, the cells were resuspended in prewarmed DMEM containing freshly prepared 2',7'-dichlorodihydrofluorescein diacetate (DCFH-DA; Beyotime Institute of Biotechnology; cat. no. S0033) according to the manufacturer's protocol. After incubation at $37^{\circ} \mathrm{C}$ for $20 \mathrm{~min}$, the cells were washed twice with DMEM and then subjected to flow cytometry analyses (Accuri C6; BD Biosciences Co., Ltd). To measure the mitochondrial ROS (mtROS) levels, treated U87-MG cells were stained with MitoSOX-Red (2 $\mu \mathrm{M}$; Molecular Probes; Thermo Fisher Scientific, Inc.) at $37^{\circ} \mathrm{C}$ for $20 \mathrm{~min}$ and washed twice with prewarmed PBS. At the end of the washing steps, the cells were analyzed via flow cytometry (Accuri C6; BD Biosciences Co., Ltd) after being collected and resuspended in prewarmed PBS as a single-cell suspension. To measure the $\Delta \Psi \mathrm{m}$, treated glioma cells were collected and resuspended as a single-cell suspension in $1 \mathrm{ml}$ culture medium, followed by incubation with the JC-1 staining solution (Beyotime Institute of Biotechnology; cat. no. C2006) for $20 \mathrm{~min}$ at $37^{\circ} \mathrm{C}$. After incubation, the cells were washed twice with JC-1 staining buffer and then analyzed via flow cytometry (Accuri C6; BD Biosciences Co., Ltd). Apoptosis was measured according to the manufacturer's protocol of the Annexin V-FITC Apoptosis Analysis kit (Tianjin Sungene Biotech Co., Ltd.; cat. no. AO2001-02P-G). Briefly, treated U87-MG cells were collected and resuspended in $1 \mathrm{ml} 1 \mathrm{X}$ binding buffer. Subsequently, $5 \mu$ Annexin V-FITC was added to the cells, followed by incubation for $10 \mathrm{~min}$ at RT in the dark. Finally, $5 \mu \mathrm{l}$ PI solution was added, followed by incubation for $5 \mathrm{~min}$ at RT. The early and late apoptotic cells were then analyzed via flow cytometry (Accuri C6; BD Biosciences Co., Ltd). The software used for flow cytometric analyses was FlowJo 10.0.7 (FlowJo LLC).

Cell Counting Kit-8 (CCK-8) assay. Cell viability was assessed using CCK-8 assays (Dojindo Molecular Technologies, Inc.; cat. no. 347-07621), which were performed in 96-well plates according to the manufacturer's protocol. Briefly, cells were added to 96-well plates and grown to $80 \%$ confluence. The CCK-8 reagent (10 $\mu \mathrm{l} /$ well) was added to glioma cells at $0,4,8$ and $24 \mathrm{~h}$ after LPS and IFN- $\gamma$ treatment. The reaction system was incubated for $2 \mathrm{~h}$ at $37^{\circ} \mathrm{C}$ in a humidified incubator with $5 \% \mathrm{CO}_{2}$, after which the absorbance readings were obtained at $450 \mathrm{~nm}$.

ELISA. The glioma cell culture supernatants were collected at $4^{\circ} \mathrm{C}$ at $106 \mathrm{x} \mathrm{g}$ for $10 \mathrm{~min}$ after LPS and IFN- $\gamma$ treatment and then to measure the secreted inflammatory mediator IL- 6 using IL-6 ELISA kits (Neobioscience; cat. no. EHC007) according to the manufacturer's protocol. Absorbance was measured at $450 \mathrm{~nm}$, and concentrations of IL-6 were calculated according to standard curves.

Statistical analysis. All experiments were repeated at least three times, and the statistical results were expressed as the mean \pm SEM. Histograms and significance were obtained with Origin 9 (OriginLab) and GraphPad Prism 5.0 (GraphPad Software, Inc.) using Student's t-test or one-way ANOVA 
A
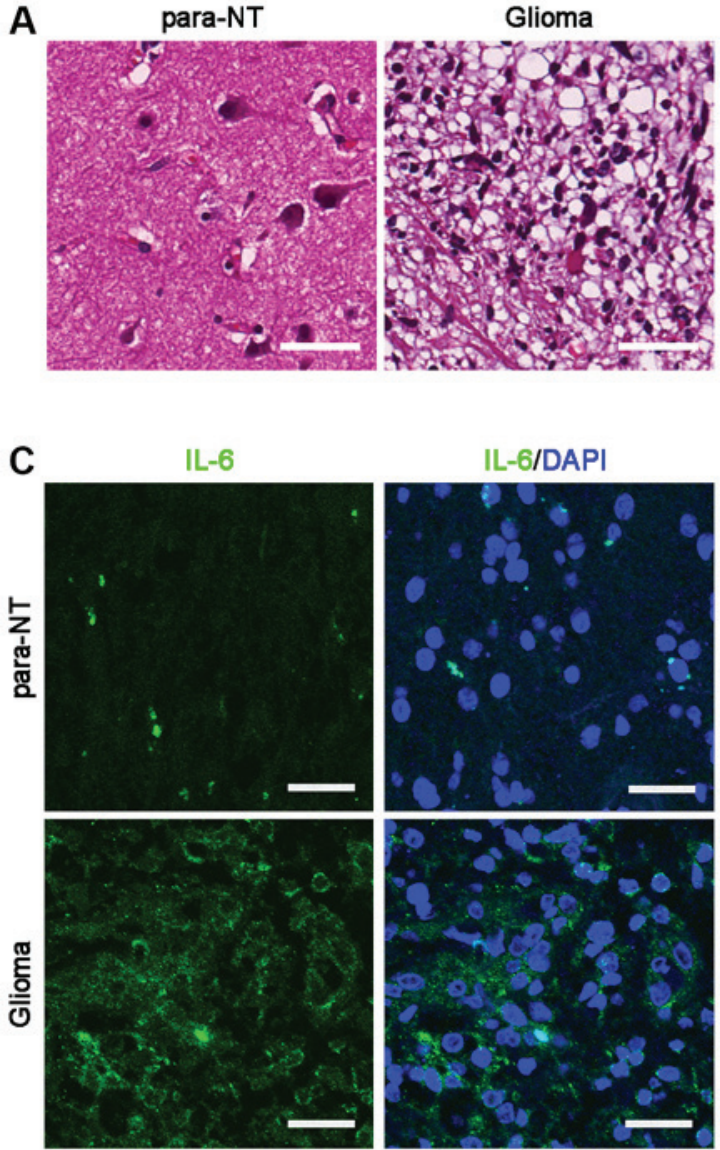

D

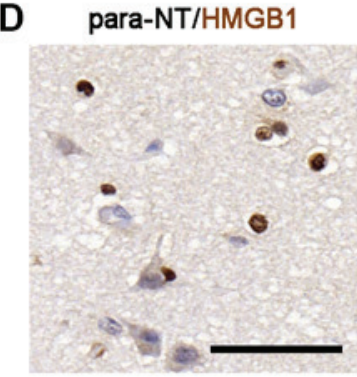

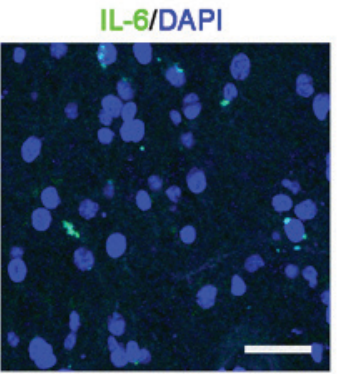

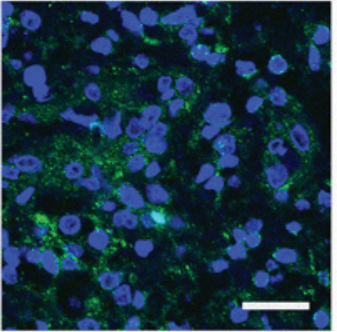

Glioma/HMGB1

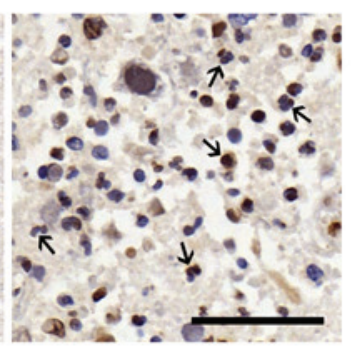

B
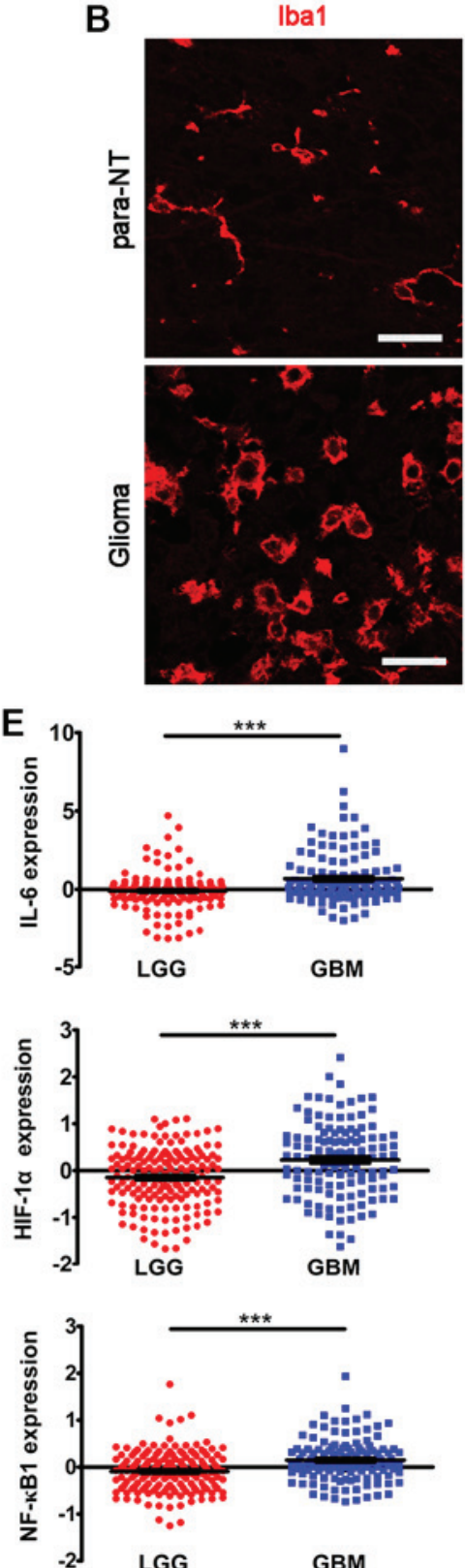
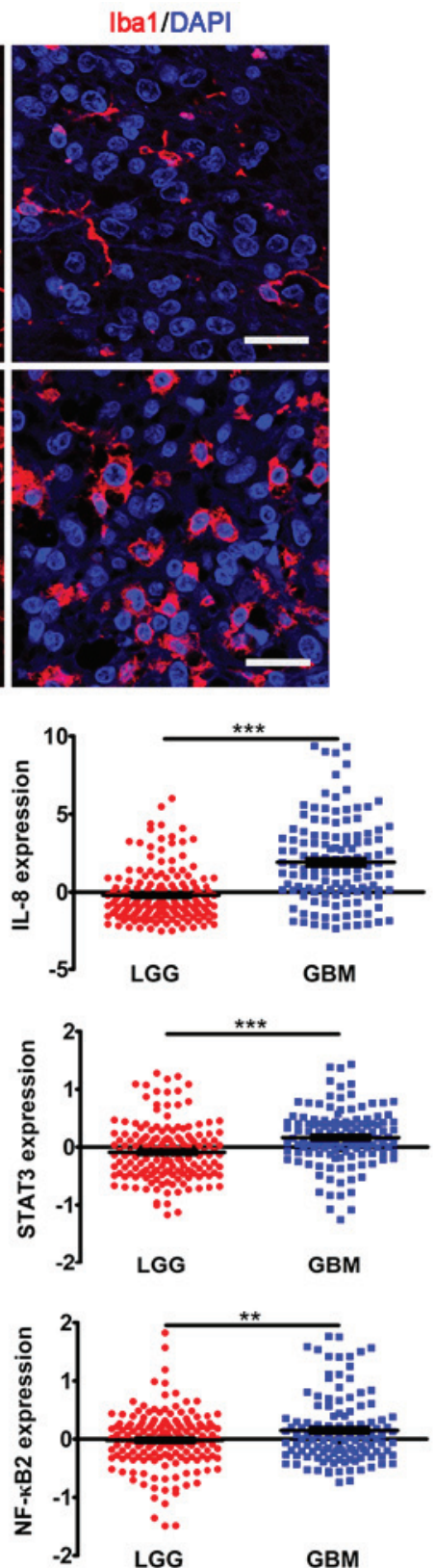

Figure 1. Inflammation in glioma. (A) Hematoxylin and eosin staining of glioma tissues and para-NTs. Scale bar, $50 \mu \mathrm{m}$.(B) IHC of Iba1 showing the microglia in glioma tissues and para-NTs. Scale bar, $25 \mu \mathrm{m}$. (C) IHC of IL-6 in glioma tissues and para-NTs. DAPI was used for nuclear visualization. Scale bar, $25 \mu \mathrm{m}$. (D) IHC of HMGB1 in glioma tissues and para-NTs. HMGB1+ inflammatory cells are indicated by black arrows. Scale bar, $50 \mu \mathrm{m}$. (E) Analyses of the mRNA expression levels of proinflammatory factors in the Chinese Glioma Genome Atlas dataset. The statistical significance was evaluated via unpaired Student's t-test. ${ }^{* *} \mathrm{P}<0.01$; ${ }^{* * *} \mathrm{P}<0.001$. para-NTs, para-neoplastic tissues; LGG, low grade glioma; GBM, glioblastoma; IHC, immunohistochemistry; HMGB1, high mobility group box 1; Iba1, ionized calcium binding adapter molecule 1; HIF-1 $\alpha$, hypoxia-inducible factor-1 $\alpha$.

followed by Tukey's multiple comparison test. Unpaired Student's t-test was used for comparisons between the normal and glioma groups, and between the LGG and GBM groups, while paired Student's t-test was used for comparisons between the para-NT and glioma groups. ANOVA was used for comparisons among multiple groups. $\mathrm{P}<0.05$ was considered to indicate a statistically significant difference.

\section{Results}

Inflammation in glioma. A major stimulus that triggers the hallmarks of cancer cells within solid tumors is the activation of inflammatory cells and the subsequent local release of proinflammatory cytokines $(2,11)$. Therefore, the present study first examined inflammatory cell activation and proinflammatory cytokine expression in glioma samples. The results revealed that locally recruited inflammatory cells and $\mathrm{Iba}^{+}$microglia were highly enriched in the glioma samples (Fig. 1A and B). Additionally, the Iba1 ${ }^{+}$processes of microglia in glioma samples were markedly shortened and the cell bodies were enlarged compared with those in para-NT samples, indicating microglia activation (Fig. 1A and B). Furthermore, the glioma tissues were highly immunoreactive with IL-6 (Fig. 1C), which is one of the major proinflammatory cytokines released from gliomas (30). Additionally, a number of $\mathrm{HMGB}^{+}$inflammatory cells were observed in 
glioma samples (Fig. 1D). It has been previously demonstrated that HMGB1 can be released by inflammatory cells into the extracellular matrix, where it participates in the inflammatory process (32). Subsequently, the gene expression data for other inflammatory factors were analyzed in the CGGA dataset. The results revealed that the expression levels of the proinflammatory cytokines $I L-6, I L-8, H I F-1 \alpha$, STAT3, $N F-\kappa B 1$ and $N F-\kappa B 2$ were significantly upregulated in GBM compared with in LGG samples (Fig. 1E). Overall, the present results demonstrated the presence of inflammation in glioma.

Defects in mitochondrial structure and metabolism in glioma. Subsequently, the mitochondrial structure in a sample from a GBM case (male; 66 years) was examined via TEM. Abnormal mitochondrial structure was observed in the glioma sample, including swelling associated with disarrangement of the cristae and partial or total cristolysis (Fig. 2A). Due to the damaged mitochondrial structure, the present study then examined whether the electron transfer chain (ETC) complexes were also affected. The expression levels of NADH dehydrogenase subunit 6, SDHB and COX6B1 were analyzed, which all participate in mitochondria oxidative phosphorylation (OXPHOS) (33-35). The IHC results revealed that, compared with in normal tissues, the levels of NADH dehydrogenase subunit 6 and SDHB in glioma tissues were significantly lower, while COX6B1 expression in glioma tissues was significantly higher compared with that in para-NTs (Fig. 2B and C). The gene expression data in the CGGA revealed that the expression levels of the glycolysis enzymes $H K 1, L D H A$ and GAPDH were significantly upregulated in GBM compared with in LGG samples (Fig. 2D). Furthermore, both the expression levels of the OXPHOS enzyme ATP synthase (ATP5A1) and the rate-limiting enzyme of the TCA cycle $C S$ were significantly downregulated in GBM compared with in LGG samples in TCGA dataset (Fig. 2E). Overall, the current results revealed an abnormal mitochondrial structure and metabolic reprogramming in glioma.

Inflammation induces mitochondrial network remodeling and mitochondrial dysfunction in glioma cells. To investigate the effect of inflammation on the mitochondrial network in glioma, glioma cells were analyzed in vitro following direct stimulation with LPS and IFN- $\gamma$, a well-established combination of factors that mimic the inflammatory response in vitro (36). The ELISA assay indicated that the secretion of IL-6, the major proinflammatory cytokines released from gliomas (30), was significantly increased at 8 and $24 \mathrm{~h}$ in U87-MG cells and at 4 , 8 and $24 \mathrm{~h}$ in U118-MG cells after the stimulation of LPS and IFN- $\gamma$ (Fig. S1), indicating the inflammatory response in glioma cells. By labeling the mitochondria with Mitotracker Red, it was revealed that the ratio of glioma cells with fragmented mitochondria was significantly increased at 4 and $8 \mathrm{~h}$ after LPS and IFN- $\gamma$ stimulation (Figs. 3A and S2Aa-f). Compared with the $0 \mathrm{~h}$ group, the ratio of cells with fragmented mitochondria in U87-MG was decreased at $24 \mathrm{~h}$ after the proinflammatory stimulation, while it was still high at $24 \mathrm{~h}$ in U118-MG cells (Figs. 3A and S2Aa-f). Notably, the fragmented mitochondria revealed spherical or ring-like morphologies after exposure to the proinflammatory stimuli (Figs. 3A and S2Aa-f). The
TEM examination revealed that these enlarged mitochondria were swollen and contained vacuoles, and that the cristae were absent (Figs. 3B and S2Ag-h). Furthermore, ring-like mitochondria were observed (Fig. 3B). The present results indicated that the fragmentation and formation of enlarged mitochondria may represent a mitochondrial stress response to inflammation stimuli.

Inflammation causes mitochondrial dysfunction in glioma cells. The aforementioned alterations in the mitochondrial dynamics strongly suggested that these alterations may contribute to the mitochondrial dysfunction in glioma. As expected, the proinflammatory stimuli caused a significant decrease in $\Delta \Psi \mathrm{m}$ in glioma cells indicated by a decrease of the ratio of red to green JC-1 fluorescence (Figs. 3C and S2B). Furthermore, the mitochondria metabolic profile was potentially affected. First, ROS measurements in stimulated U87-MG cells were performed. DCFH-DA and MitoSOX-Red were used to examine the ceROS and mtROS levels, respectively. After $4 \mathrm{~h}$ of treatment with LPS and IFN- $\gamma$, the levels of ceROS and mtROS production were significantly increased compared with those in non-stimulated glioma cells, and the high ROS levels persisted until $8 \mathrm{~h}$ after treatment; notably, mtROS production decreased at $24 \mathrm{~h}$, but ceROS production was unchanged (Fig. 4A and B). Therefore, proinflammatory stimuli may lead to transient ROS production by mitochondria as they undergo structural disruption in U87-MG cells. ROS levels in U118-MG cells were also evaluated, but there was no statistically significant difference (data not shown). The spherical or ring-like mitochondria in the present experiment were similar to those in oxidatively damaged cultured cardiomyocytes (37). Due to this similarity, the present study next examined whether the mitochondrial membrane proteins and lipids were oxidized in glioma cells. Notably, ICC staining with an antibody to $\mathrm{HNE}$, which recognizes oxidized proteins and lipids, did not specifically colocalize with the mitochondria, indicating that there was no oxidative damage of the mitochondrial membrane after the stimulation with LPS and IFN- $\gamma$ (Figs. 4C and S3). Subsequently, the mitochondrial metabolic profile was analyzed, revealing that stimulated U87-MG glioma cells exhibited marked increases in their expression levels of HK1 and LDHA, which are both glycolysis enzymes (Fig. 4D). By contrast, the expression levels of the ETC complexes COX6B1 and SDHB were significantly downregulated in cells after $8 \mathrm{~h}$ of stimulation, consistent with OXPHOS alterations (Fig. 4D). However, the expression levels of the rate-limiting enzyme of the TCA cycle CS were significantly upregulated in the stimulated glioma cells, which was inconsistent with the bioinformatics results, indicating a possible discrimination between glioma cells in vitro and glioma tissues (Fig. 4D). Notably, the mitochondrial metabolic profile in U118-MG cells was not significantly changed after proinflammatory stimuli (data not shown). Overall, the current results revealed that inflammation may lead to defective mitochondrial function and metabolic reprogramming in glioma cells in vitro.

Dysfunctional mitochondria are not cleared via mitophagy. Considering the nature of the mitochondrial defects observed following inflammatory stimuli, the present study focused on 
A

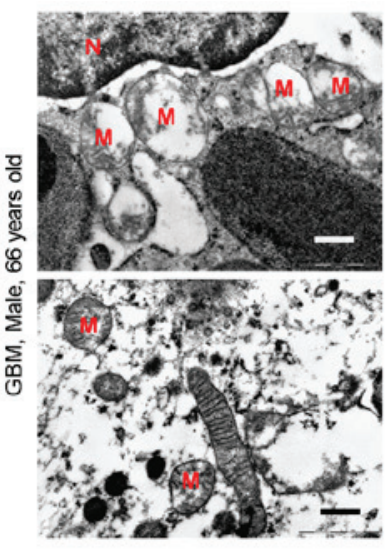

B

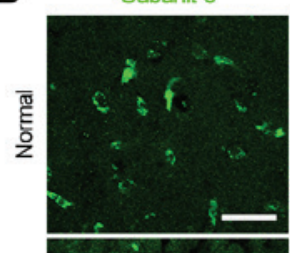

Subunit 6/DAPI

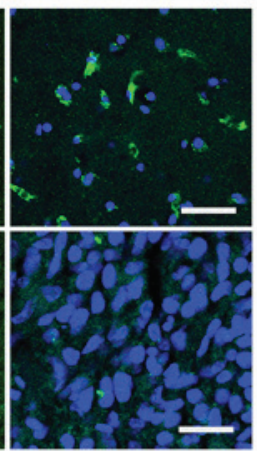

SDHB

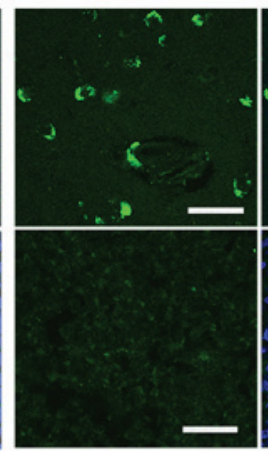

SDHB/DAPI

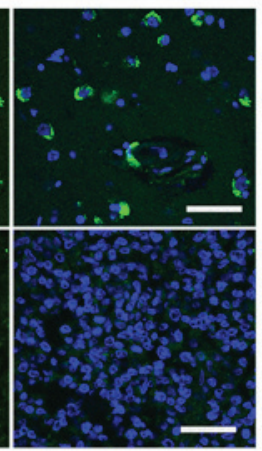

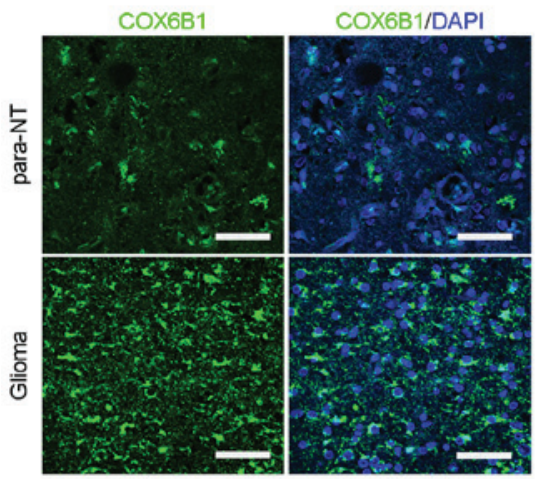

C
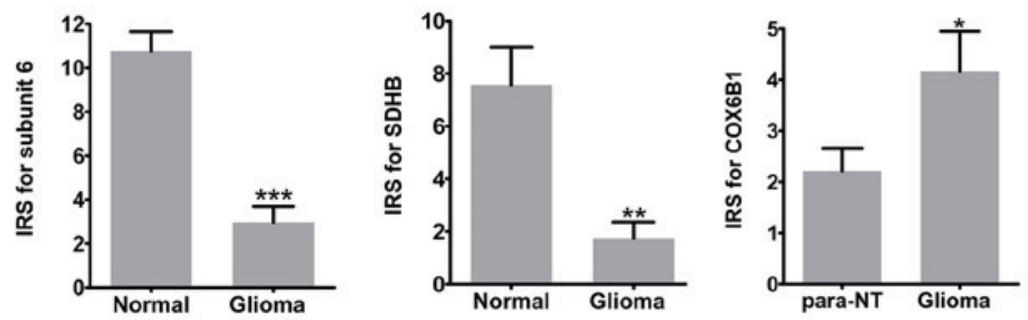

D
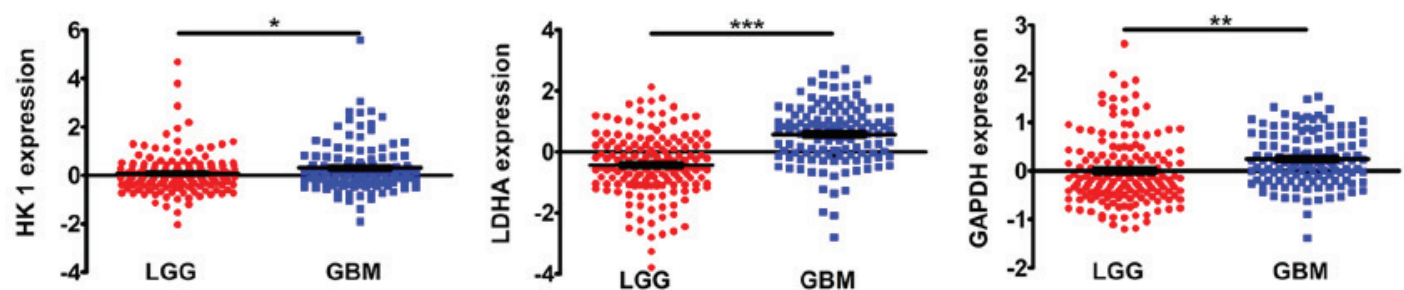

E
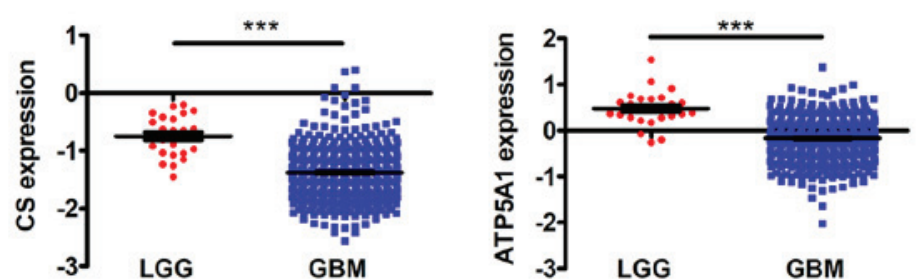

Figure 2. Mitochondrial defects in glioma. (A) Representative transmission electron microscopy images of a case of GBM (male, 66 years). Scale bar, $500 \mathrm{~nm}$. (B) Immunohistochemistry of subunit 6, SDHB and COX6B1 in glioma tissues and normal or para-NTs. DAPI was used for nuclear visualization. Scale bar, $25 \mu \mathrm{m}$. (C) Histograms of the statistical analyses of the IRSs of subunit 6, SDHB and COX6B1. (D) Expression levels of HK1, LDHA and GAPDH in the Chinese Glioma Genome Atlas. (E) Expression levels of CS and ATP5A1 in The Cancer Genome Atlas. The statistical significance was evaluated via unpaired Student's t-test, except for COX6B1 (paired Student's t-test). ${ }^{*} \mathrm{P}<0.05 ;{ }^{* *} \mathrm{P}<0.01 ;{ }^{* * *} \mathrm{P}<0.001$. Subunit 6, NADH dehydrogenase subunit 6; M, mitochondria; N, nucleus; GBM, glioblastoma; LGG, low-grade glioma; para-NTs, para-neoplastic tissues; IRS, immunoreactive score; SDHB, iron-sulfur protein subunit of succinate dehydrogenase; COX6B1, cytochrome C oxidase subunit VIb; HK1, hexokinase 1; CS, citrate synthase; LDHA, lactate dehydrogenase A; ATP5A1, ATP synthase.

possible mechanisms that may mediate its resolution. One of the possibilities may involve the clearance of the damaged mitochondria via autophagy, a form of quality control considered to be important for maintaining the functionality of mitochondrial networks known as mitophagy $(36,38)$. Therefore, the present study examined whether autophagy was induced following exposure to proinflammatory stimuli by evaluating the lipidation of the autophagy-associated protein $\mathrm{LC} 3 \mathrm{~B}$. LC3B abundance and its conversion from the cytosolic isoform LC3B-I to the autophagosomal-associated isoform LC3B-II was examined in glioma cells via ICC and western blotting. The results revealed that the proinflammatory stimuli caused a significant increase in LC3B puncta and LC3B-II abundance in U87-MG cells (Fig. 5A, C, E and F), but not in U118-MG cells (Fig. 5B, D, G and H). However, there was no detectable change in mitophagy (determined as the colocalization of mitochondria with the LC3B positive signals) at any time point in both glioma cells. A similar result was observed after transfecting a GFP-LC3 plasmid into the U87-MG cells (Fig. S4A). To further track the fate of 
A
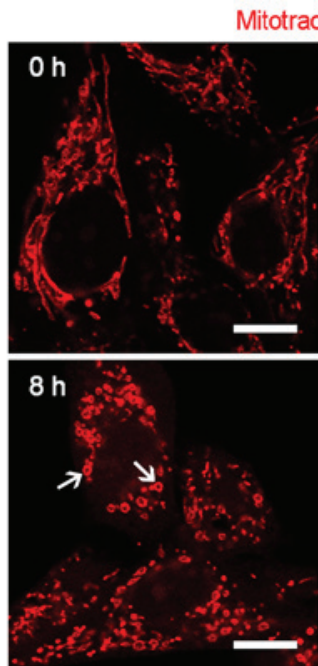

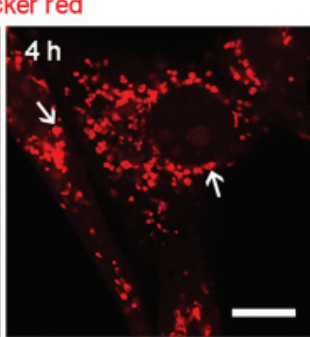

$24 \mathrm{~h}$

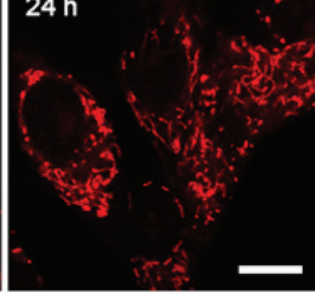

B Non-stimulated

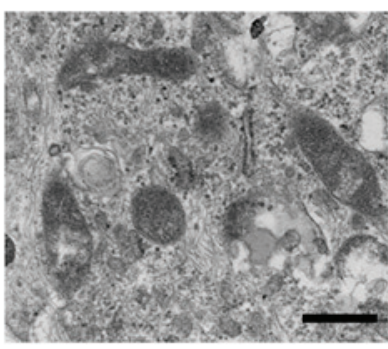

C
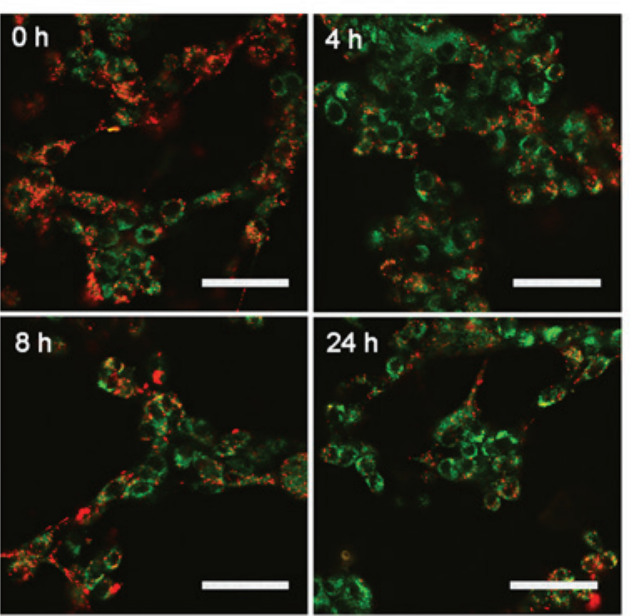

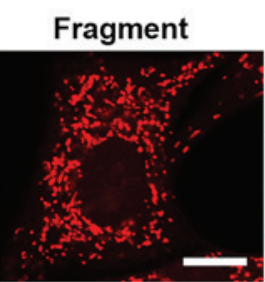

Intermediate
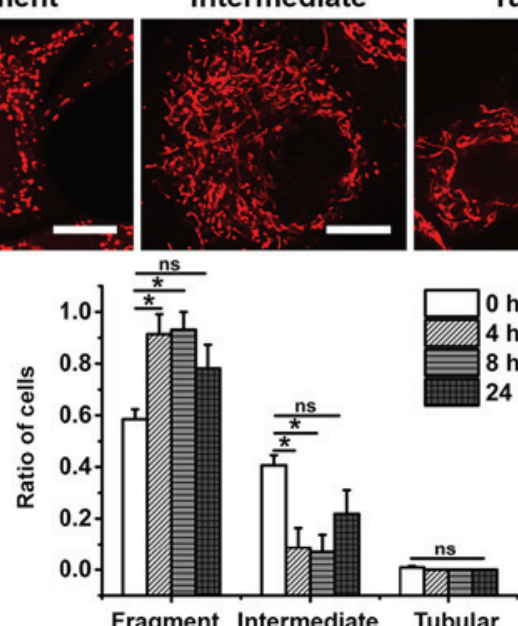

LPS+IFN- $y$

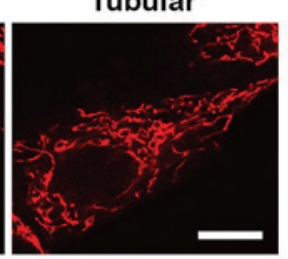

o h

h

$24 \mathrm{~h}$
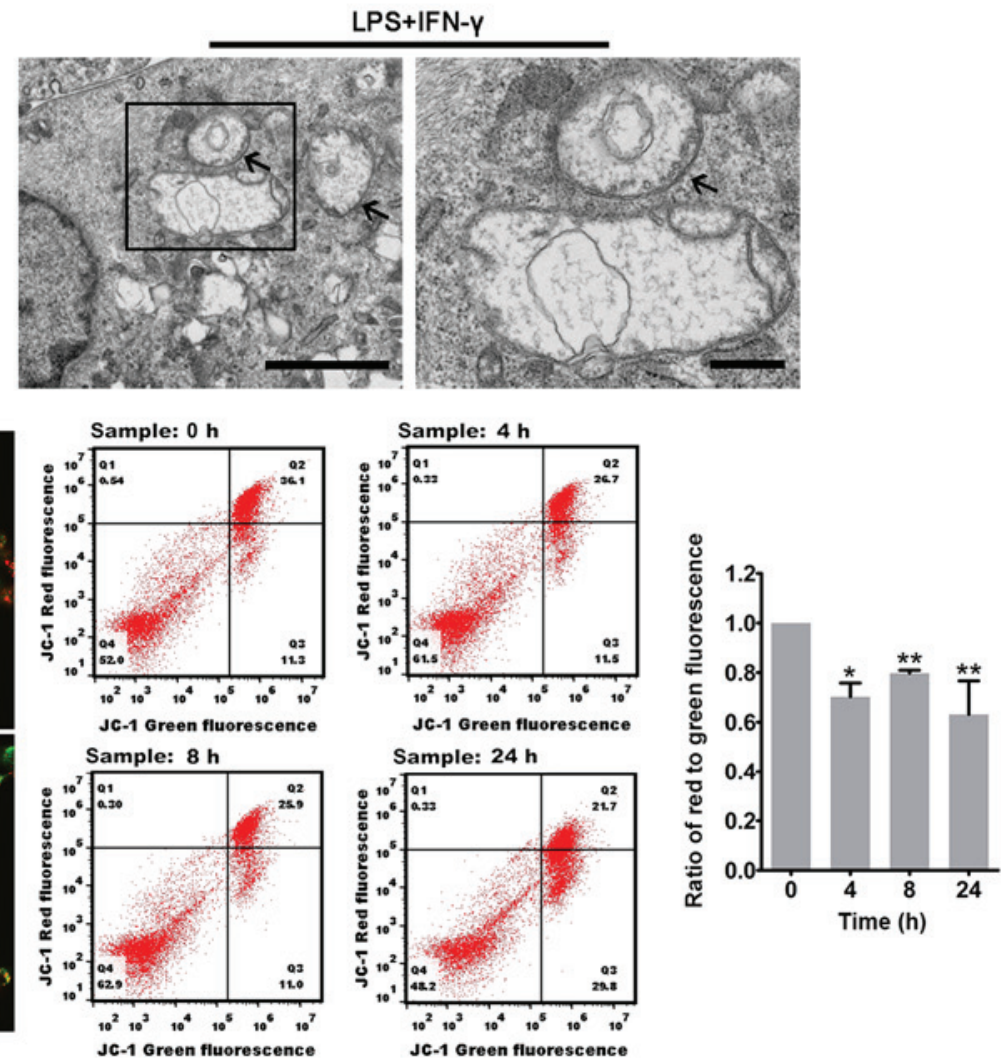

Figure 3. Proinflammatory stimuli induce mitochondrial network remodeling in glioma cells. (A) Representative images of mitochondria in U87-MG cells at different time points after LPS and IFN- $\gamma$ stimulation. Mitotracker Red served as the mitochondrial probe. The white arrows indicate the spherical or ring-like mitochondria. The histogram represents the statistical analyses of the ratios of glioma cells with fragmented, intermediate or tubular mitochondria ( $=4$; 50-150 cells per time point). The upper right three images are representative of glioma cells with fragmented, intermediate or tubular mitochondria. Scale bar, $10 \mu \mathrm{m}$. (B) Representative transmission electron microscopy images of mitochondria in U87-MG cells before (non-stimulated; left panel; scale bar, 500 nm) and after LPS and IFN- $\gamma$ treatment (middle panel; scale bar, $2 \mu \mathrm{m}$ ). The black arrows indicate the ring-like mitochondria. The rectangular area was enlarged in the right panel (scale bar, $500 \mathrm{~nm}$ ). (C) Fluorescent images were acquired via confocal microscopy after U87-MG cells were treated with the JC-1 staining solution (scale bar, $50 \mu \mathrm{m}$ ). A change from red to green reflects decreased $\Delta \Psi \mathrm{m}$, which was also analyzed via flow cytometry. The histogram presents the statistical analysis of the $\Delta \Psi \mathrm{m}$ after LPS and IFN- $\gamma$ treatment $(\mathrm{n}=3)$. The statistical significance was evaluated via one-way ANOVA followed by Tukey's post hoc test. ${ }^{*} \mathrm{P}<0.05$; ${ }^{* *} \mathrm{P}<0.01$; ns, not significant; LPS, lipopolysaccharide; $\Delta \Psi \mathrm{m}$, mitochondrial membrane potential; fluor, fluorescence.

the dysfunctional mitochondria, the current study explored whether they could also interact with acidic compartments. However, no distinct colocalization of mitochondria with the lysosomal marker LAMP1 was observed after exposure to the proinflammatory stimuli in U87-MG cells (Fig. S4B). The present results indicated that the dysfunctional mitochondria resulting from the proinflammatory stimuli were not being cleared via mitophagy. Finally, the effect of the persistence of the damaged mitochondria on glioma cell survival was examined. The results revealed that exposure to the proinflammatory stimuli markedly decreased the apoptosis of U87-MG cells (Fig. 6A and B), while the apoptosis of U118-MG cells was not influenced by the proinflammatory stimuli (data not shown). Additionally, the 

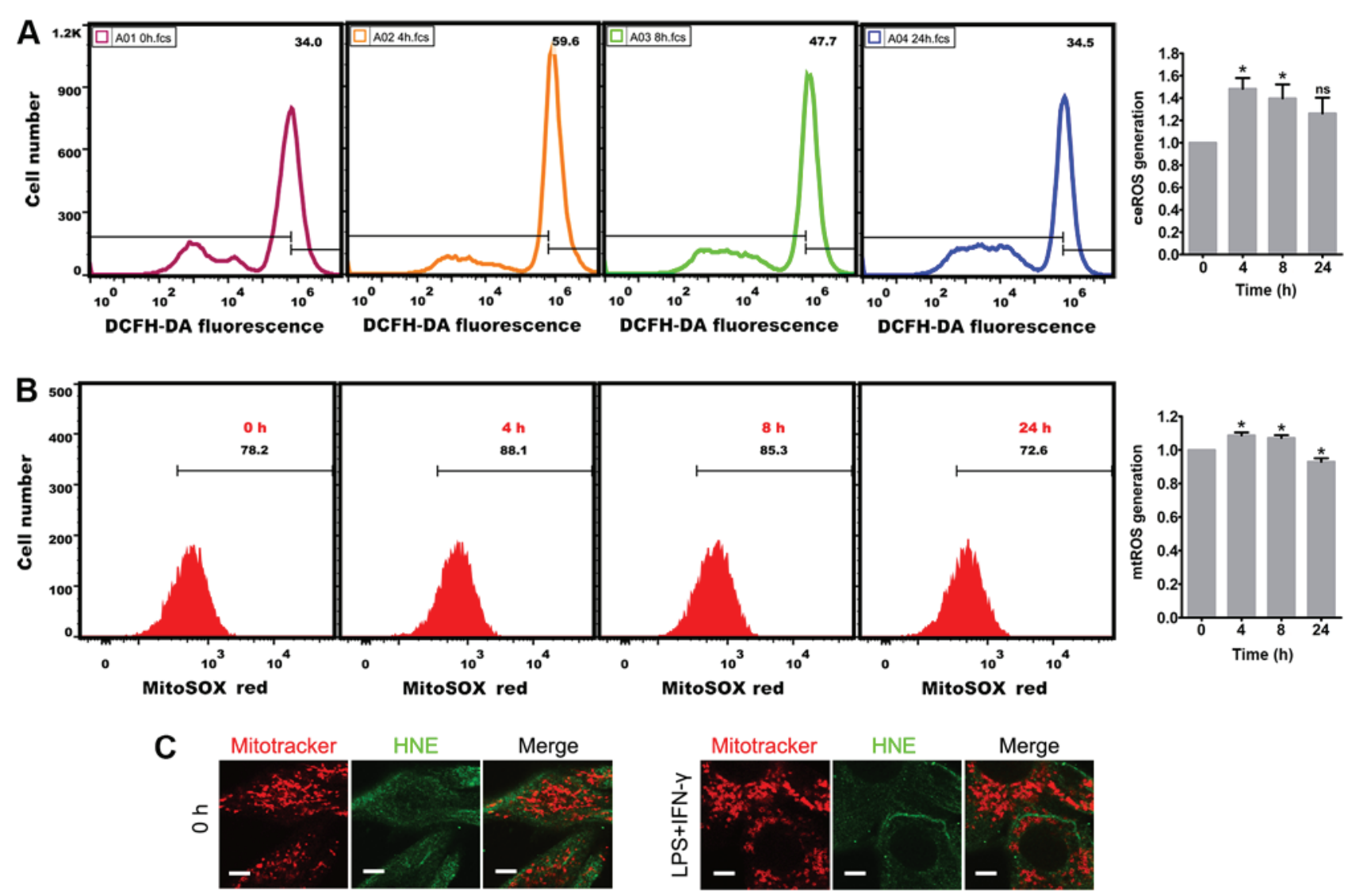

D

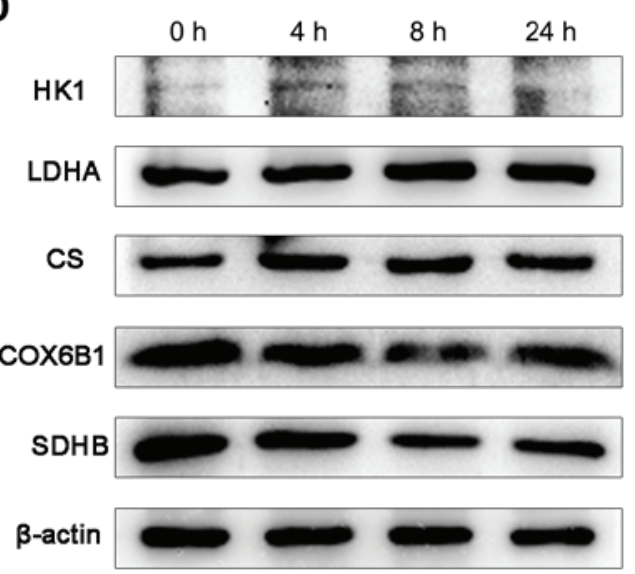

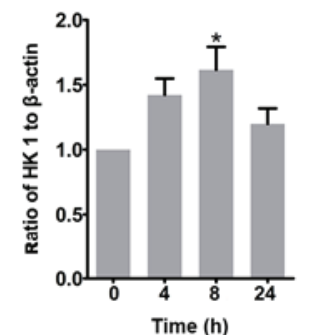
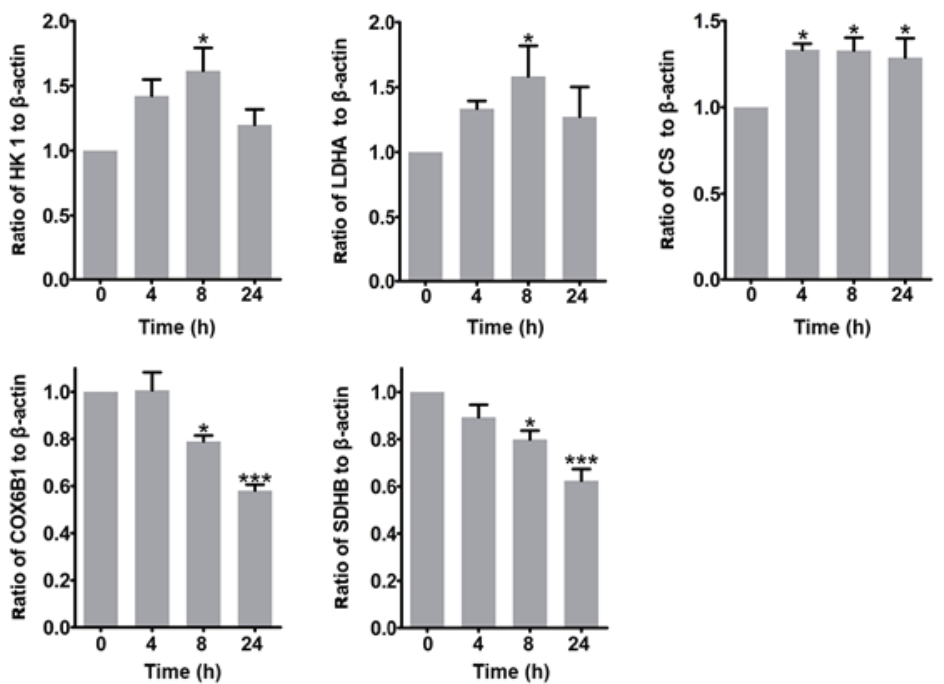

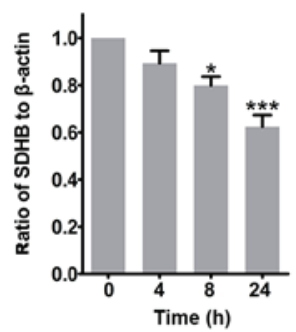

Figure 4. Proinflammatory stimuli induce ROS generation and metabolic remodeling in glioma cells. (A) Total ceROS in U87-MG cells was measured by staining with DCFH-DA and flow cytometry. The histogram presents the statistical analysis of ceROS generation after LPS and IFN- $\gamma$ treatment ( $\mathrm{n}=5$ ). (B) mtROS in U87-MG cells was measured by staining with MitoSOX-Red and flow cytometry. The histogram presents the statistical analysis of mtROS generation after LPS and IFN- $\gamma$ treatment $(\mathrm{n}=4)$. (C) Double-labeling of U87-MG cells with Mitotracker Red and HNE. Scale bar, $5 \mu \mathrm{m}$. (D) Protein expression levels of HK1, LDHA, CS, COX6B1 and SDHB in control $(0 \mathrm{~h})$ and LPS and IFN- $\gamma$-treated U87-MG cells. The histograms show the statistical analyses of the ratios of HK1, LDHA, CS, COX6B1 and SDHB to $\beta$-actin (n=3). The statistical significance was evaluated via one-way ANOVA followed by Tukey's post hoc test. " $\mathrm{P}<0.05$; ${ }^{* * *} \mathrm{P}<0.001$; ns, not significant; LPS, lipopolysaccharide: ROS; reactive oxygen species; ceROS, cellular ROS; mtROS, mitochondrial ROS; HNE, 4-hydroxynonenal; DCFH-DA, 2',7'-dichlorodihydrofluorescein diacetate; SDHB, iron-sulfur protein subunit of succinate dehydrogenase; COX6B1, cytochrome C oxidase subunit VIb; HK1, hexokinase 1; CS, citrate synthase; LDHA, lactate dehydrogenase A.

CCK-8 assay demonstrated that the viability of glioma cells was not affected by the mitochondrial dysfunction caused by the proinflammatory stimuli (Fig. 6C and D). Overall, the aforementioned results demonstrated that inflammation increased the tolerance of glioma cells to dysfunctional mitochondria.

\section{Discussion}

Inflammation is a common condition that occurs in solid tumors due to the activation and recruitment of local and circulating proinflammatory cells, and secretion of inflammatory factors (11). In the present study, recruitment of inflammatory 

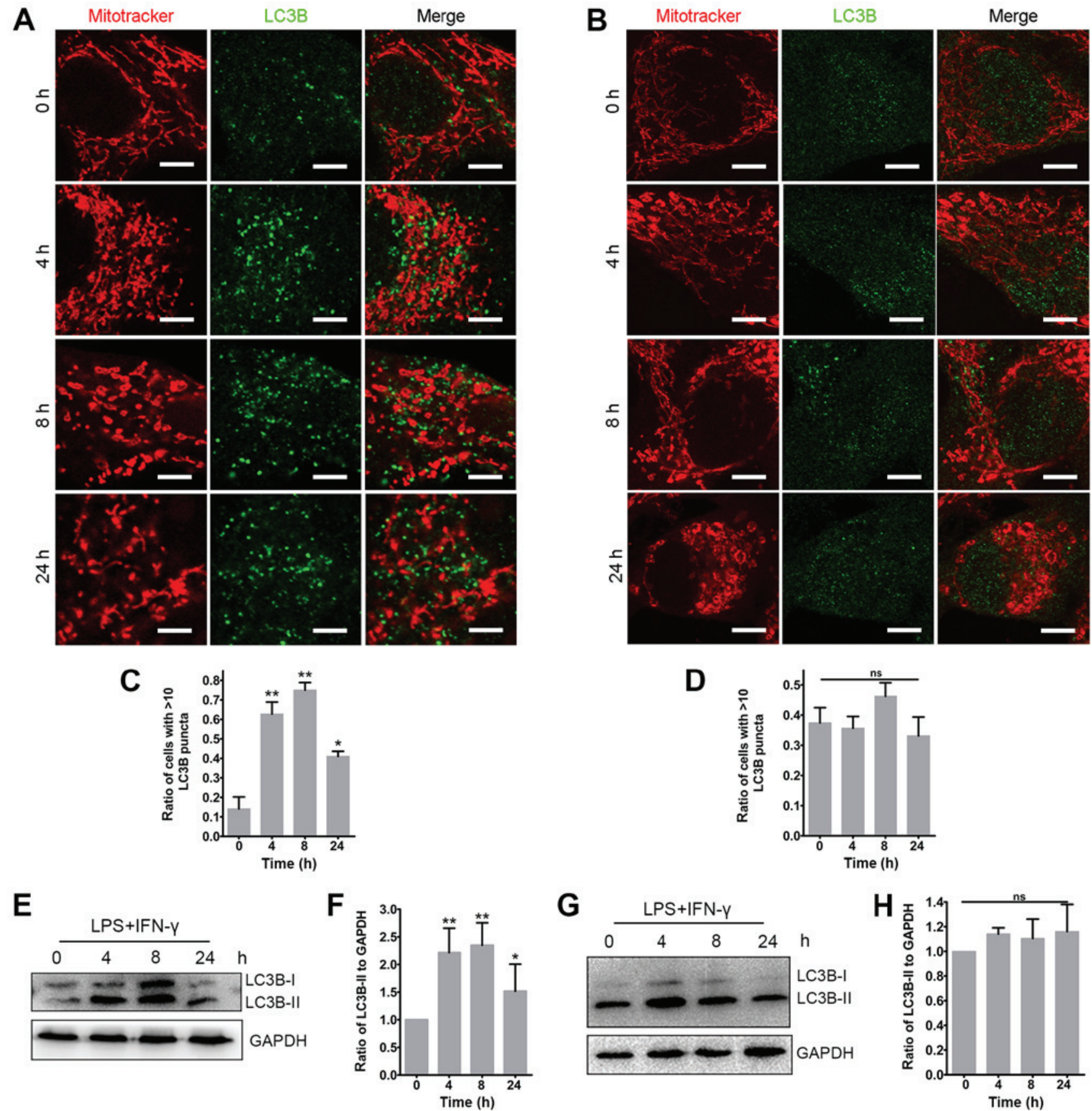

Figure 5. Dysfunctional mitochondria are not cleared via mitophagy. Expression levels of LC3B in (A) U87-MG and (B) U118-MG cells before (0 h) and after LPS and IFN- $\gamma$ stimulation. Scale bar, $5 \mu \mathrm{m}$. Scale bar, $10 \mu \mathrm{m}$. Statistical analyses of the ratio of cells with $>10$ LC3B puncta in (C) U87-MG and (D) U118-MG cells ( $\mathrm{n}=4 ; 50-150$ cells per time point). (E) Western blot analysis of the autophagosomal-associated isoform LC3B-II expression in U87-MG cells. (F) Ratios of LC3B-II to GAPDH ( $\mathrm{n}=3$ ). (G) Western blot analysis of the autophagosomal-associated isoform LC3B-II expression in U118-MG cells. (H) Ratios of LC3B-II to GAPDH (n=3). Mitotracker Red was used to detect the mitochondria. The statistical significance was evaluated via one-way ANOVA followed by Tukey's post hoc test. ${ }^{*} \mathrm{P}<0.05 ;{ }^{* *} \mathrm{P}<0.01$; ns, not significant; LPS, lipopolysaccharide.

cells, microglial reactions and upregulation of proinflammatory factors were observed in glioma tissue samples, indicating the presence of an inflammatory inner environment in glioma. Mitochondrial dysfunction in tumors has been associated with abnormalities in mitochondrial energy metabolism, marked by a metabolic shift from oxidative phosphorylation to glycolysis (known as the 'Warburg effect'), disturbances in $\Delta \Psi \mathrm{m}$ regulation and apoptotic signaling (21). The present experiments demonstrated that mitochondrial network remodeling manifested as swelling and damaged cristae and altered expression levels of ETC complexes in glioma. In addition, upregulation of glycolytic enzymes and downregulation of OXPHOS enzymes were observed in glioma. The current results provide strong evidence for the presence of mitochondrial dysfunction and metabolic reprogramming in glioma, which has been reviewed by Strickland and Stoll (21).

To address the influence of inflammation on the mitochondria in glioma cells, the present study examined how the mitochondrial dynamics changed in glioma cells directly exposed to proinflammatory stimuli in vitro. Stimulation 


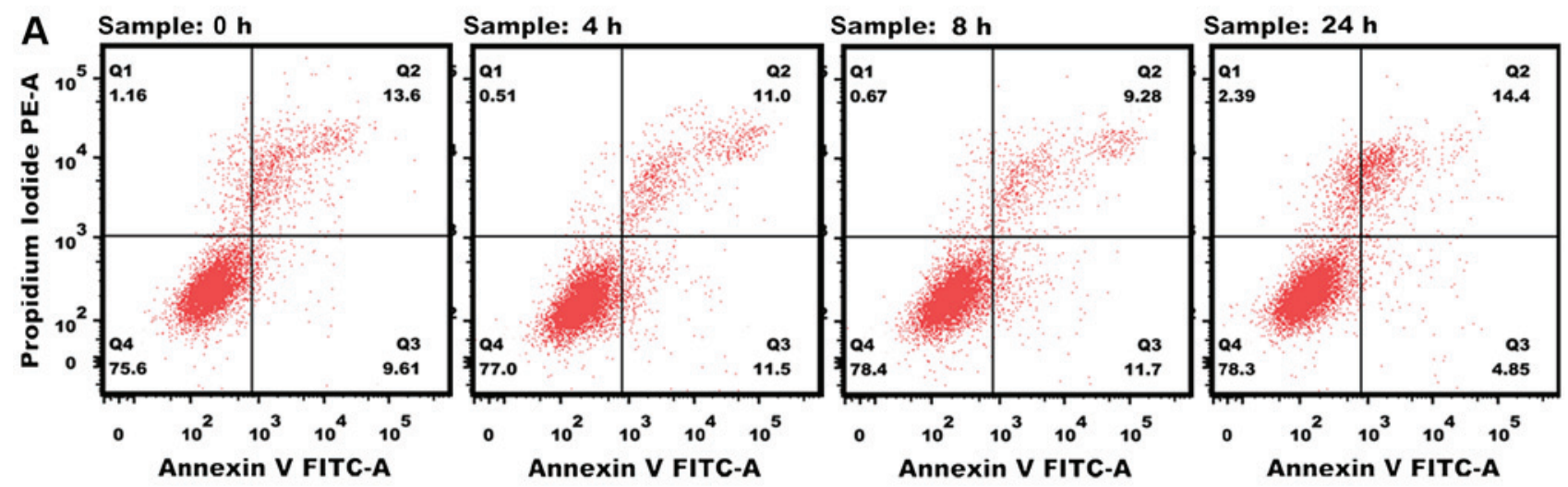

B

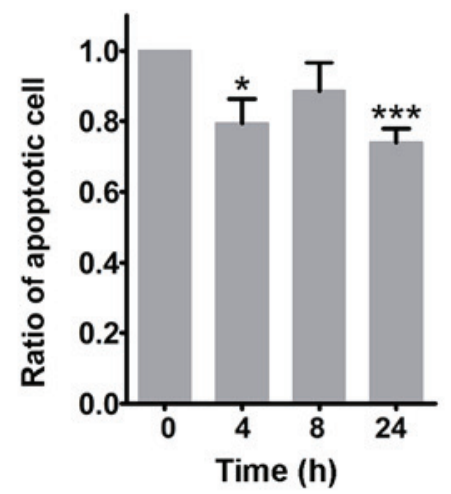

C

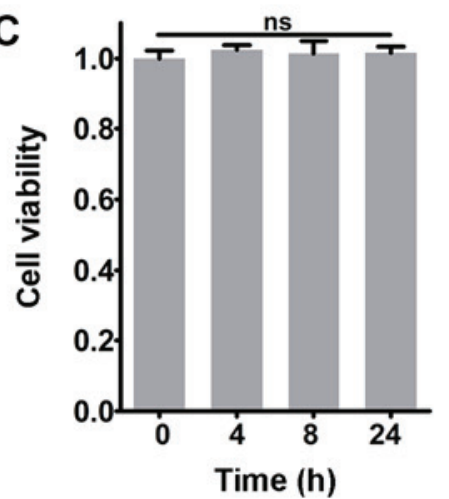

D

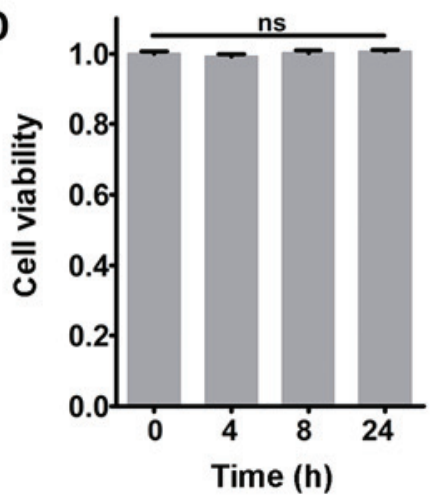

Figure 6. Glioma cells are resistant to the mitochondrial dysfunction. (A) Apoptosis of U87-MG cells was examined via flow cytometric analyses of Annexin V-stained cells. (B) Statistical analyses of apoptosis before $(0 \mathrm{~h})$ and after LPS and IFN- $\gamma$ treatment ( $\mathrm{n}=4)$. Cell Counting Kit- 8 assays of the viability of (C) U87-MG and (D) U118-MG cells before ( $0 \mathrm{~h})$ and after LPS and IFN- $\gamma$ treatment $(\mathrm{n}=4)$. The statistical significance was evaluated via one-way ANOVA followed by Tukey's post hoc test. ${ }^{*} \mathrm{P}<0.05$; ${ }^{* * *} \mathrm{P}<0.001$; ns, not significant; LPS, lipopolysaccharide.

with LPS and IFN- $\gamma$ is a well-established combination of factors that mimic the inflammatory response in vitro (36). In our previous study, glioma cells were stimulated with $1 \mu \mathrm{g} / \mathrm{ml}$ LPS and $10 \mathrm{ng} / \mathrm{ml}$ IFN- $\gamma$ (36); however, no marked changes were observed in the mitochondria of glioma cells. Therefore, the concentrations of LPS and IFN- $\gamma$ were increased in the present study, revealing that a combination of $4 \mu \mathrm{g} / \mathrm{ml}$ LPS and $40 \mathrm{ng} / \mathrm{ml} \mathrm{IFN}-\gamma$ was able to induce a significant remodeling of the mitochondrial network. Additionally, this combination of LPS and IFN- $\gamma$ induced the secretion of the inflammatory cytokine IL- 6 in glioma cells, which is one of the major proinflammatory cytokines released from gliomas (30). The current results revealed that proinflammatory stimuli induced rapid and profound changes in the mitochondrial network, leading to its fragmentation, the appearance of spherical or ring-like morphologies, and damaged ultrastructure in glioma cells. This structural disruption of the mitochondria resulted in a significant decrease in the $\Delta \Psi \mathrm{m}$, indicating impaired mitochondria function after exposure to inflammatory stimuli. Mitochondria are the main cellular source of ROS (39). Numerous studies have investigated the roles of ROS as a tumor-promoting or tumor-suppressing agent, and there is abundant evidence supporting both possibilities (25,40-44). In the present study, significant increases in both the ceROS and mtROS levels in glioma cells were detected after 4 and $8 \mathrm{~h}$ of treatment. By contrast, these levels were lower at $24 \mathrm{~h}$, consistent with a partial recovery of the mitochondrial network. The HNE ICC results revealed that ROS did not induce oxidative damage to the mitochondrial membranes, indicating that the mitochondria can tolerate the ROS induced by inflammation in glioma cells. Furthermore, the mitochondrial network remodeling may reveal forms of mitochondrial plasticity that are important for adjusting the metabolic state of the glioma to cope with the metabolic challenges induced by inflammatory stress. Indeed, the levels of the glycolytic enzymes HK1 and LDHA were substantially increased, while the levels of the ETC complexes COX6B1 and SDHB were decreased. Notably, the levels of CS, the rate-limiting enzyme in the TCA cycle, were significantly increased in proinflammatory-stimulated glioma cells. Previous studies have demonstrated an aberrant function of the TCA cycle in cancer $(45,46)$. CS catalyzes the first committed step of the TCA cycle and its expression has been reported to be upregulated in several types of cancer (47-49). For example, upregulated CS expression has been associated with cell proliferation, invasion and migration in human ovarian carcinoma (47). Consistently, the upregulation of CS expression observed in the present study may reflect a type of metabolic reprogramming in inflammation-stimulated glioma cells. In light of the widely accepted belief that cancer cells primarily utilize aerobic glycolysis, the role of the TCA cycle in glioma metabolism and tumorigenesis should be further investigated. In addition, COX6B1 

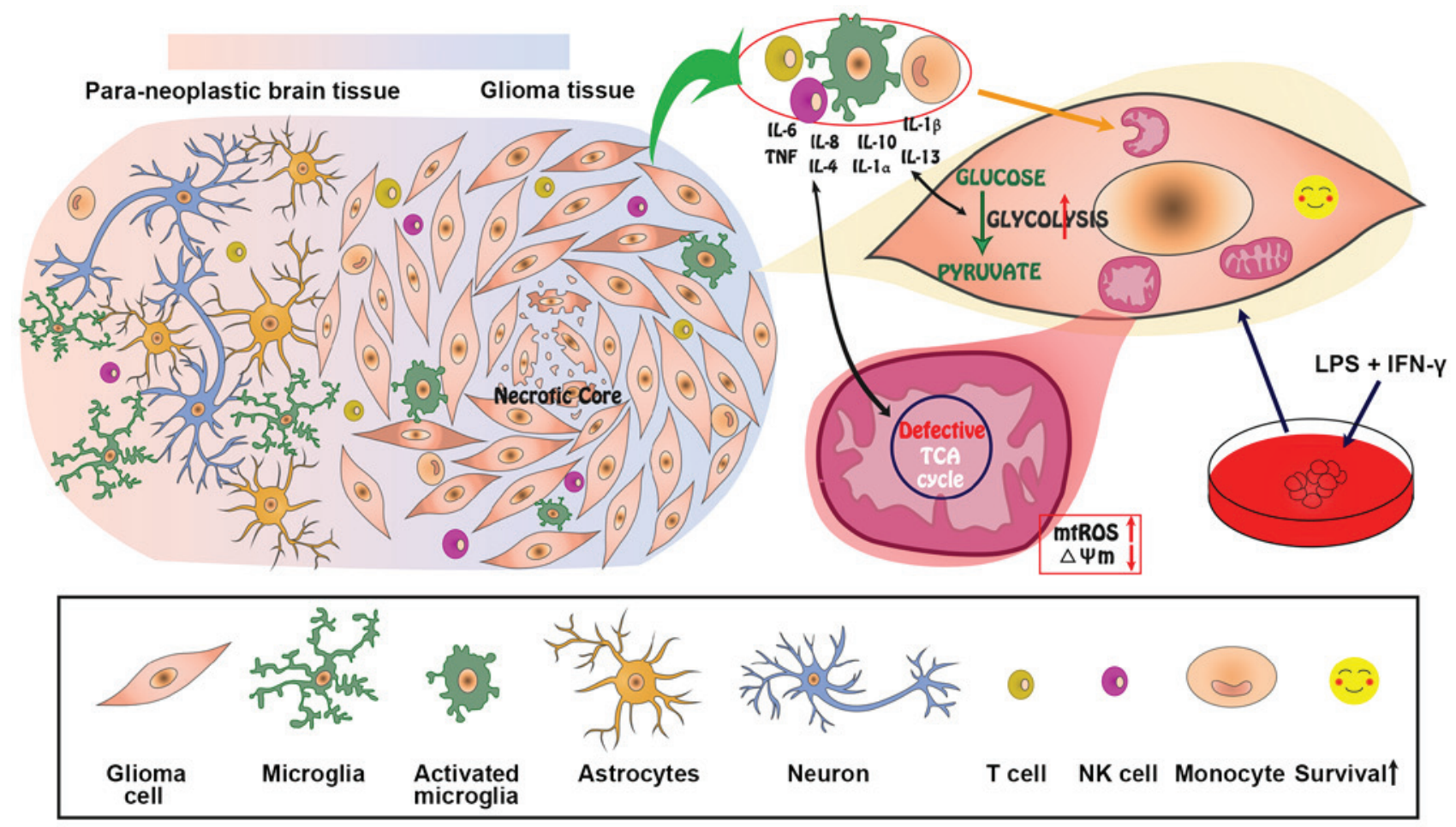

Figure 7. Model and hypothesis for the effect of inflammation on the mitochondrial dynamics in glioma. Inflammation induced by the infiltration of inflammatory cells and secretion of proinflammatory factors may cause mitochondrial fragmentation, swelling, vacuolation, disrupted $\Delta \Psi \mathrm{m}$, and increased ROS levels. Additionally, the mitochondrial metabolism may be remodeled by inflammation, resulting in increased glycolysis and a defective TCA cycle. The dysfunctional mitochondria are not cleared via mitophagy, but rather persist in the glioma cells to improve their survival. $\Delta \Psi$ m, mitochondrial membrane potential; ROS, reactive oxygen species; mtROS, mitochondrial ROS; TCA, tricarboxylic acid; HIF-1 $\alpha$, hypoxia-inducible factor-1 $\alpha$; LPS, lipopolysaccharide; $\mathrm{NK}$, natural killer.

expression was observed to be upregulated in glioma tissues compared with in para-NT. As a cytochrome oxidase subunit, COX6B1 is encoded in the nuclear genome and serves critical roles in energy metabolism and regulation (50). It has been demonstrated that COX6B1 overexpression inhibits apoptosis and induces mitochondrial respiration and stress resistance $(35,51)$. Accordingly, COX6B1 upregulation in glioma may be a response of the glioma cells to increase their resistance to a harsh environment. However, the in vitro results indicated the opposite, since COX6B1 expression was downregulated after 8 and $24 \mathrm{~h}$ of exposure to proinflammatory stimuli. It was speculated that this effect may be due to differences between in vitro and in vivo conditions. The tumor microenvironment is more complicated than that under in vitro conditions; therefore, the increased levels of COX6B1 in glioma may result from a number of unknown factors. From this perspective, the in vitro results may more accurately reflect the effects of inflammation on mitochondria in glioma cells.

An important mechanism of mitochondria clearance is mitophagy, which has been reported in several studies (52-54). Mitochondrial fragmentation and a decline in the $\Delta \Psi \mathrm{m}$ are essential conditions for mitophagy (55-57). In the present study, the mitochondrial network was partially recovered $24 \mathrm{~h}$ after treatment, as was the production of ceROS and mtROS. However, no marked alteration in the colocalization of Mitotracker Red and LC3B (indicating mitophagy) was observed at any point. Furthermore, the dysfunctional mitochondria were not engulfed by lysosomes. Therefore, the present results suggested that the damaged mitochondria induced by inflammation were not cleared via mitophagy. Future studies should investigate how abnormal mitochondria induced by inflammatory stress achieve effective quality control. A possibility may be that these mitochondria are not 'bad enough' that they must be cleared and are 'salvageable; thus, the mitochondria activate the relevant self-help mechanisms to normalize the network (58). It has been reported that reduction or ablation of the $\Delta \Psi \mathrm{m}$ can induce nuclear translocation of activating transcription factor associated with stress-1, where it initiates the mitochondrial unfolded protein response, which is a mitochondria self-help mechanism (59). Whether the mitochondria observed in the present study initiated this self-help mechanism remains to be further investigated. Additionally, it is possible that the formation of spherical or ring-like mitochondrial structures may reflect a response to environmental conditions that have functional consequences. Mitochondria gradually adapt to the inflammatory environment, becoming more tolerant to inflammation and less sensitive to the environmental conditions $(60,61)$. Therefore, the fragmented, spherical or ring-like mitochondria gradually recovered, and the altered mitochondrial morphology may represent a response to inflammatory stress. Furthermore, in certain stages of tumorigenesis, decreased mitophagy may allow for a permissive threshold for the persistence of dysfunctional mitochondria, which then generate increased tumor-promoting ROS or other tumorigenic mitochondrial signals (25). Accordingly, a decrease in apoptosis was observed in the present study; therefore, the persistence of 
dysfunctional mitochondria may help glioma cells to survive in inflammatory environments.

However, there are some limitations in the present study. For example, the lack of normalization of the data downloaded from the CGGA dataset generated negative values during the statistical analysis. Additionally, isocitrate dehydrogenase mutation status may dramatically affect the function of mitochondria (62). Therefore, the lack of mutation experiments is another potential limitation. Furthermore, the expression levels of proteins (such as IL-6, IL-8, HIF-1 $\alpha$, STAT3, NF- $\kappa$ B1 and $\mathrm{NF}-\kappa \mathrm{B} 2$ ) in the inflammatory microenvironment in glioma should be further evaluated using IHC staining or western blotting.

In conclusion, the current results identified a direct association between inflammation and changes in mitochondrial dynamics in glioma, revealing that inflammation may induce mitochondrial dysfunction in glioma, including fragmentation, swelling, structural and $\Delta \Psi \mathrm{m}$ disruption, and ROS generation. Finally, metabolic reprogramming may result in increased aerobic glycolysis, a defective TCA cycle and OXPHOS (Fig. 7). Although the recovery or biogenesis of the mitochondria, and the role of the TCA cycle in glioma after proinflammatory stimuli should be further investigated, the present results demonstrated that the dysfunction of these organelles did not affect the viability but decreased the apoptosis of glioma cells (Fig. 7). Further insight into how inflammatory processes impact local bioenergetics within glioma tissues may lead to the identification of novel routes for understanding the association between inflammation and solid tumors.

\section{Acknowledgements}

The authors would like to sincerely thank Professor Shuping Zhang (State Key Laboratory of Biomembrane and Membrane Biotechnology, School of Life Sciences, Tsinghua University, Beijing, China) for providing the GFP-LC3 constructs.

\section{Funding}

The present study was supported by the National Natural Science Foundation of China (grant no. 81402455) and the Key Scientific Research Projects of Higher Education Institutions in Henan Province (grant no. 20A310020). The funding sources had no involvement in the design of the study and collection, analysis and interpretation of data, and in writing the manuscript.

\section{Availability of data and materials}

All data generated or analyzed during this study are included in this published article.

\section{Authors' contributions}

LJ acquired funding. WF, XC, ZR, YS, PL and HS acquired the data. LJ, WF, XC, ZR and YS analyzed and interpreted the data. WF, XC, ZR and YS statistically analyzed the data. LJ wrote the manuscript. All authors read and approved the final manuscript.

\section{Ethics approval and consent to participate}

Life Science Ethics Committee of Zhengzhou University (Zhengzhou, China) reviewed and approved the study according to the principles expressed in the Declaration of Helsinki. Written informed consent was provided by each participant or their families.

\section{Patient consent for publication}

Not applicable.

\section{Competing interests}

The authors declare that they have no competing interests.

\section{References}

1. Ostrom QT, Gittleman H, Xu J, Kromer C, Wolinsky Y, Kruchko C and Barnholtz-Sloan JS: CBTRUS statistical report: Primary brain and other central nervous system tumors diagnosed in the United States in 2009-2013. Neuro Oncol 18 (Suppl 5): v1-v75, 2016.

2. Conti A, Gulì C, La Torre D, Tomasello C, Angileri FF and Aguennouz M: Role of inflammation and oxidative stress mediators in gliomas. Cancers (Basel) 2: 693-712, 2010.

3. Ham SW, Jeon HY, Jin X, Kim EJ, Kim JK, Shin YJ, Lee Y, Kim SH, Lee SY, Seo S, et al: TP53 gain-of-function mutation promotes inflammation in glioblastoma. Cell Death Differ 26: 409-425, 2019

4. Philip M, Rowley DA and Schreiber H: Inflammation as a tumor promoter in cancer induction. Semin Cancer Biol 14: 433-439, 2004.

5. Aggarwal BB, Vijayalekshmi RV and Sung B: Targeting inflammatory pathways for prevention and therapy of cancer: Short-term friend, long-term foe. Clin Cancer Res 15: 425-430, 2009.

6. Grivennikov SI and Karin M: Inflammation and oncogenesis: A vicious connection. Curr Opin Genet Dev 20: 65-71, 2010.

7. Samadi AK, Bilsland A, Georgakilas AG, Amedei A, Amin A, Bishayee A, Azmi AS, Lokeshwar BL, Grue B, Panis C, et al: A multi-targeted approach to suppress tumor-promoting inflammation. Semin Cancer Biol 35: S151-S184, 2015.

8. Mantovani A, Allavena P, Sica A and Balkwill F: Cancer-related inflammation. Nature 454: 436-444, 2008.

9. Colotta F, Allavena P, Sica A, Garlanda C and Mantovani A: Cancer-related inflammation, the seventh hallmark of cancer: Links to genetic instability. Carcinogenesis 30: 1073-1081, 2009.

10. Hussain SP, Hofseth LJ and Harris CC: Radical causes of cancer. Nat Rev Cancer 3: 276-285, 2003.

11. Schetter AJ, Heegaard NH and Harris CC: Inflammation and cancer: Interweaving microRNA, free radical, cytokine and p53 pathways. Carcinogenesis 31: 37-49, 2010

12. Kembro JM, Cortassa S, Lloyd D, Sollott SJ and Aon MA: Mitochondrial chaotic dynamics: Redox-energetic behavior at the edge of stability. Sci Rep 8: 15422, 2018.

13. Blajszczak $\mathrm{C}$ and Bonini MG: Mitochondria targeting by environmental stressors: Implications for redox cellular signaling. Toxicology 391: 84-89, 2017.

14. Altieri DC: Mitochondria on the move: Emerging paradigms of organelle trafficking in tumour plasticity and metastasis. $\mathrm{Br}$ J Cancer 117: 301-305, 2017.

15. Zhai K, Chang L, Zhang Q, Liu B and Wu Y: Mitochondrial C150T polymorphism increases the risk of cervical cancer and HPV infection. Mitochondrion 11: 559-563, 2011.

16. Canter JA, Kallianpur AR, Parl FF and Millikan RC: Mitochondrial DNA G10398A polymorphism and invasive breast cancer in African-American women. Cancer Res 65: 8028-8033, 2005.

17. Permuth-Wey J, Chen YA, Tsai YY, Chen Z, Qu X, Lancaster JM, Stockwell H, Dagne G, Iversen E, Risch H, et al: Inherited variants in mitochondrial biogenesis genes may influence epithelial ovarian cancer risk. Cancer Epidemiol Biomarkers Prev 20: 1131-1145, 2011.

18. Katsetos CD, Anni H and Draber P: Mitochondrial dysfunction in gliomas. Semin Pediatr Neurol 20: 216-227, 2013. 
19. Arismendi-Morillo GJ and Castellano-Ramirez AV: Ultrastructural mitochondrial pathology in human astrocytic tumors: Potentials implications pro-therapeutics strategies. J Electron Microsc (Tokyo) 57: 33-39, 2008.

20. Guntuku L, Naidu VG and Yerra VG: Mitochondrial dysfunction in gliomas: Pharmacotherapeutic potential of natural compounds. Curr Neuropharmacol 14: 567-583, 2016.

21. Strickland M and Stoll EA: Metabolic reprogramming in glioma. Front Cell Dev Biol 5: 43, 2017.

22. Waitkus MS, Diplas BH and Yan H: Isocitrate dehydrogenase mutations in gliomas. Neuro Oncol 18: 16-26, 2016.

23. Warburg O: On the origin of cancer cells. Science 123: 309-314, 1956.

24. Modica-Napolitano JS and Weissig V: Treatment strategies that enhance the efficacy and selectivity of mitochondria-targeted anticancer agents. Int J Mol Sci 16: 17394-17421, 2015.

25. Vyas S, Zaganjor E and Haigis MC: Mitochondria and cancer. Cell 166: 555-566, 2016.

26. Mancias JD and Kimmelman AC: Mechanisms of selective autophagy in normal physiology and cancer. J Mol Biol 428: $1659-1680,2016$

27. Sun H, Zhang M, Cheng K, Li P, Han S, Li R, Su M, Zeng W, Liu J, Guo J, et al: Resistance of glioma cells to nutrient-deprived microenvironment can be enhanced by CD133-mediated autophagy. Oncotarget 7: 76238-76249, 2016.

28. Isakovic AM, Dulovic M, Markovic I, Kravic-Stevovic T, Bumbasirevic V, Trajkovic V and Isakovic A: Autophagy suppression sensitizes glioma cells to IMP dehydrogenase inhibition-induced apoptotic death. Exp Cell Res 350: 32-40, 2017.

29. Louis DN, Perry A, Reifenberger G, von Deimling A, Figarella-Branger D, Cavenee WK, Ohgaki H, Wiestler OD, Kleihues P and Ellison DW: The 2016 world health organization classification of tumors of the central nervous system: A summary. Acta Neuropathol 131: 803-820, 2016

30. Xue H, Yuan G, Guo X, Liu Q, Zhang J, Gao X, Guo X, Xu S, $\mathrm{Li}$ T, Shao Q, et al: A novel tumor-promoting mechanism of IL6 and the therapeutic efficacy of tocilizumab: Hypoxia-induced IL6 is a potent autophagy initiator in glioblastoma via the p-STAT3-MIR155-3p-CREBRF pathway. Autophagy 12: $1129-1152,2016$

31. Jia L, Liang T, Yu X, Ma C and Zhang S: MGARP regulates mouse neocortical development via mitochondrial positioning. Mol Neurobiol 49: 1293-1308, 2014.

32. Weber DJ, Allette YM, Wilkes DS and White FA: The HMGB1-RAGE inflammatory pathway: Implications for brain injury-induced pulmonary dysfunction. Antioxid Redox Signal 23: 1316-1328, 2015.

33. Bai Y and Attardi G: The mtDNA-encoded ND6 subunit of mitochondrial NADH dehydrogenase is essential for the assembly of the membrane arm and the respiratory function of the enzyme. EMBO J 17: 4848-4858, 1998.

34. Wang H, Luo J, Tian W, Yan W, Ge S, Zhang Y and Sun W: $\gamma$-tocotrienol inhibits oxidative phosphorylation and triggers apoptosis by inhibiting mitochondrial complex I subunit NDUFB8 and complex II subunit SDHB. Toxicology 417: 42-53, 2019.

35. Kim SE, Mori R, Komatsu T, Chiba T, Hayashi H, Park S, Sugawa MD, Dencher NA and Shimokawa I: Upregulation of cytochrome c oxidase subunit $6 \mathrm{~b} 1$ (Cox6b1) and formation of mitochondrial supercomplexes: Implication of Cox6b1 in the effect of calorie restriction. Age (Dordr) 37: 9787, 2015.

36. Motori E, Puyal J, Toni N, Ghanem A, Angeloni C, Malaguti M, Cantelli-Forti G, Berninger B, Conzelmann KK, Götz M, et al: Inflammation-induced alteration of astrocyte mitochondrial dynamics requires autophagy for mitochondrial network maintenance. Cell Metab 18: 844-859, 2013.

37. Kageyama Y,Hoshijima M, Seo K, Bedja D, Sysa-Shah P,Andrabi SA, Chen W,Höke A, Dawson VL, Dawson TM, et al: Parkin-independent mitophagy requires Drpl and maintains the integrity of mammalian heart and brain. EMBO J 33: 2798-2813, 2014

38. Williams JA and Ding WX: Mechanisms, pathophysiological roles and methods for analyzing mitophagy-recent insights. Biol Chem 399: 147-178, 2018

39. Dan Dunn J, Alvarez LA, Zhang $X$ and Soldati T: Reactive oxygen species and mitochondria: A nexus of cellular homeostasis. Redox Biol 6: 472-485, 2015.
40. Chio IIC and Tuveson DA: ROS in cancer: The burning question. Trends Mol Med 23: 411-429, 2017.

41. Shadel GS and Horvath TL: Mitochondrial ROS signaling in organismal homeostasis. Cell 163: 560-569, 2015.

42. Sullivan LB and Chandel NS: Mitochondrial reactive oxygen species and cancer. Cancer Metab 2: 17, 2014

43. Jin L, Li D, Alesi GN, Fan J, Kang HB, Lu Z, Boggon TJ, Jin P, Yi H, Wright ER, et al: Glutamate dehydrogenase 1 signals through antioxidant glutathione peroxidase 1 to regulate redox homeostasis and tumor growth. Cancer Cell 27: 257-270, 2015.

44. DeNicola GM, Karreth FA, Humpton TJ, Gopinathan A, Wei C, Frese K, Mangal D, Yu KH, Yeo CJ, Calhoun ES, et al: Oncogene-induced Nrf2 transcription promotes ROS detoxification and tumorigenesis. Nature 475: 106-109, 2011.

45. Gaude E and Frezza C: Defects in mitochondrial metabolism and cancer. Cancer Metab 2: 10, 2014.

46. Anderson NM, Mucka P, Kern JG and Feng H: The emerging role and targetability of the TCA cycle in cancer metabolism. Protein Cell 9: 216-237, 2018.

47. Chen L, Liu T, Zhou J, Wang Y, Wang X, Di W and Zhang S: Citrate synthase expression affects tumor phenotype and drug resistance in human ovarian carcinoma. PLoS One 9: e115708, 2014.

48. Schlichtholz B, Turyn J, Goyke E, Biernacki M, Jaskiewicz K, Sledzinski Z and Swierczynski J: Enhanced citrate synthase activity in human pancreatic cancer. Pancreas 30: 99-104, 2005.

49. Lin CC, Cheng TL, Tsai WH, Tsai HJ, Hu KH, Chang HC, Yeh CW, Chen YC, Liao CC and Chang WT: Loss of the respiratory enzyme citrate synthase directly links the Warburg effect to tumor malignancy. Sci Rep 2: 785, 2012.

50. Sinkler CA, Kalpage H, Shay J, Lee I, Malek MH, Grossman LI and Hüttemann M: Tissue- and condition-specific isoforms of mammalian cytochrome c oxidase subunits: From function to human disease. Oxid Med Cell Longev 2017: 1534056, 2017.

51. Zhang W, Wang Y, Wan J, Zhang P and Pei F: COX6B1 relieves hypoxia/reoxygenation injury of neonatal rat cardiomyocytes by regulating mitochondrial function. Biotechnol Lett 41: 59-68, 2019.

52. Um JH and Yun J: Emerging role of mitophagy in human diseases and physiology. BMB Rep 50: 299-307, 2017.

53. Kulikov AV, Luchkina EA, Gogvadze V and Zhivotovsky B: Mitophagy: Link to cancer development and therapy. Biochem Biophys Res Commun 482: 432-439, 2017.

54. Ney PA: Mitochondrial autophagy: Origins, significance, and role of BNIP3 and NIX. Biochim Biophys Acta 1853: 2775-2783, 2015.

55. Arnoult D, Rismanchi N, Grodet A, Roberts RG, Seeburg DP, Estaquier J, Sheng M and Blackstone C: Bax/Bak-dependent release of DDP/TIMM8a promotes Drp1-mediated mitochondrial fission and mitoptosis during programmed cell death. Curr Biol 15: 2112-2118, 2005

56. Dengjel $\mathrm{J}$ and Abeliovich $\mathrm{H}$ : Roles of mitophagy in cellular physiology and development. Cell Tissue Res 367: 95-109, 2017.

57. Eiyama A and Okamoto K: PINK1/Parkin-mediated mitophagy in mammalian cells. Curr Opin Cell Biol 33: 95-101, 2015.

58. Kornmann B: Quality control in mitochondria: Use it, break it, fix it, trash it. F1000Prime Rep 6: 15, 2014.

59. Deng P and Haynes CM: Mitochondrial dysfunction in cancer: Potential roles of ATF5 and the mitochondrial UPR. Semin Cancer Biol 47: 43-49, 2017.

60. Ding WX, Li M, Biazik JM, Morgan DG, Guo F, Ni HM, Goheen M, Eskelinen EL and Yin XM: Electron microscopic analysis of a spherical mitochondrial structure. J Biol Chem 287: 42373-42378, 2012.

61. Ding WX, Guo FL, Ni HM, Bockus A, Manley S, Stolz DB, Eskelinen EL, Jaeschke H and Yin XM: Parkin and mitofusins reciprocally regulate mitophagy and mitochondrial spheroid formation. J Biol Chem 287: 42379-42388, 2012.

62. Findlay AS, Carter RN, Starbuck B, McKie L, Nováková K, Budd PS, Keighren MA, Marsh JA, Cross SH, Simon MM, et al: Mouse Idh3a mutations cause retinal degeneration and reduced mitochondrial function. Dis Model Mech 11: 2018.

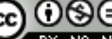

This work is licensed under a Creative Commons Attribution-NonCommercial-NoDerivatives 4.0 International (CC BY-NC-ND 4.0) License. 